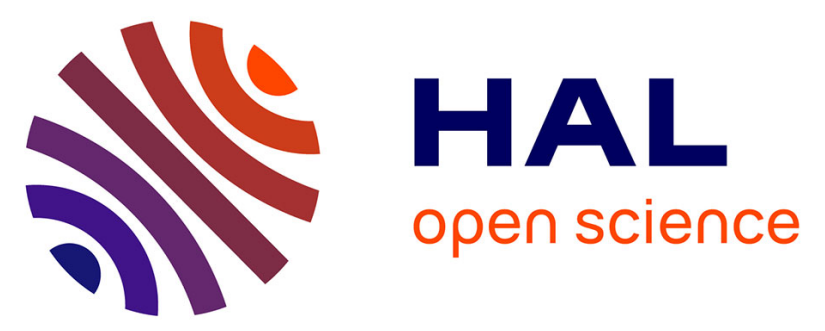

\title{
Nonlinear growth of the converging Richtmyer-Meshkov instability in a conventional shock tube
}

Marc Vandenboomgaerde, Pascal Rouzier, Denis Souffland, Laurent Biamino, Georges Jourdan, Lazhar Houas, Christian Mariani

\section{- To cite this version:}

Marc Vandenboomgaerde, Pascal Rouzier, Denis Souffland, Laurent Biamino, Georges Jourdan, et al. Nonlinear growth of the converging Richtmyer-Meshkov instability in a conventional shock tube. Physical Review Fluids, 2018, 3 (1), 10.1103/PhysRevFluids.3.014001 . hal-02080772

\section{HAL Id: hal-02080772 \\ https://hal-amu.archives-ouvertes.fr/hal-02080772}

Submitted on 27 Mar 2019

HAL is a multi-disciplinary open access archive for the deposit and dissemination of scientific research documents, whether they are published or not. The documents may come from teaching and research institutions in France or abroad, or from public or private research centers.
L'archive ouverte pluridisciplinaire HAL, est destinée au dépôt et à la diffusion de documents scientifiques de niveau recherche, publiés ou non, émanant des établissements d'enseignement et de recherche français ou étrangers, des laboratoires publics ou privés. 


\title{
Nonlinear growth of the converging Richtmyer-Meshkov instability in a conventional shock tube.
}

\author{
Marc Vandenboomgaerde, Pascal Rouzier, and Denis Souffland \\ CEA, DAM, DIF, F-91297 Arpajon, France
}

\author{
Laurent Biamino, Georges Jourdan, Lazhar Houas, and Christian Mariani \\ IUSTI, Aix Marseille Université, 5 rue Enrico Fermi 13013 Marseille
}

(Dated: June 22, 2017)

\section{INTRODUCTION}

Converging shock waves are involved in several fieds of high energy physics such as astrophysics and inertial confinement fusion (ICF). As their strength increases with time, these focusing shock waves can efficiently heat and compress matter. In the wake of these shock waves, the matter implodes and further compresses. However, the efficiency of this compression can be tempered if the symmetry of the flow is broken. The Richtmyer-Meshkov (RM) hydrodynamics instabilities [1, 2] can disrupt this symmetry. It is induced by the passage of a shock wave through a density gradient. For example, in ICF experiments, such gradient occurs at the interface between the deuteriumtritium (DT) core, and the encapsulating spherical plastic shell. A specific sequence of converging shock waves is launched to compress the DT fuel. If defects are present at the DT/plastic interface, the RM instability will be triggered. The amplitude of the defect increases, and it could lead to a mixing between the core and the plastic. A mixing zone within the DT fuel would damp the nuclear reactions within the core. The control of the RM instabilities is crucial to ICF success, and reliable models which predict the growth of the RM instabilities in convergent geometries are needed.

In the past few decades, the RM instability was often studied in planar geometry. Numerous models have been derived in the planar geometry to describe the linear and the nonlinear regimes of the instability for compressible and incompressible flows. Their reliability have been assessed by comparison with lots of experiments which were often performed in planar shock tubes. In convergent geometry, the growth of the RM instability can be enhanced. In order to test the corresponding theories and numerical simulations, experimental results with controlled initial conditions are needed. Such experiments with perfect gases are scarce [3]. A few more data are available with laser experiments [4-7]. These experiments are extremely elaborated [8, 9] and required heavy facilities [10-12]. Furthermore, high energy physics must be taken into account in order to explain the experimental data [13]. Some models have been derived to study the RM instability in convergent geometry for the canonical problem of a single mode sinusoidal perturbation between two perfect gases $[16,17,19,20]$. They do not take into account neither plasma, radiation, thermal transport or ablation physics. Nonetheless, they are an essential step in order to assess the distinction between the incompressible convergence $[14,15]$, the acceleration or deceleration of the interface which leads to the Rayleigh-Taylor (RT) instability [21, 22], and the compressibility. Furthermore, nonlinear theories are also needed [23], and must be tested in the case of a convergent geometry. In the following, we present the results of experiments about the canonical cylindrical RM instability which starts from the linear regime to the reach the nonlinear stage.

Since 2014 [24, 25], we have built a new shock tube facility which generates cylindrical RM instabilities. We study this instability at the interface between perfect gases ( $\mathrm{SF}_{6}$ and air) which are seperated by a single-mode sinusoidal interface. This shock tube is obtained from a conventional planar shock tube by using the gas lens technique [26]. This technique turns a planar shock wave into a convergent one through the impedance mismatch at a first shaped interface. The resulting shock wave is then guided toward a second interface where the RM instability is studied. The interfaces between gases are materialized by thin nitrocellulosic membranes which are held on shaped stereolithographed grids. In the following, we present and analyze the experimental results which are obtained with such a facility.

The paper is organized as follows. In Sec. II, we present the experimental set-up. In Sec. III, the 1D flows are discussed. This leads us to specify the actual composition of the involved gases. In Sec IV, we compare the experimental and numerical shapes of the interface where the RM instability takes place. We explain how the imprint due to the grid bars on the initial shape of the interface must be taken into account. The different features of the experimental data are specified. In Sec. V, we focus on the growth of the instability. Experimental and numerical peak-to-valley amplitudes are compared. A good agreement is obtained. A theoretical analysis of these cylindrical RM instabilities is also presented. We show that the interface is not purely coasting after the shock passage, and it undergoes a slight deceleration before being shocked again (re-shock). As a consequence, the interface is subject to a 
Rayleigh-Taylor instability which initial conditions are determined by the Richtmyer-Meshkov instability. Finally, we will demonstrate that the growth of the instability does not saturate in the nonlinear regime as it would have in the planar case.

\section{EXPERIMENTAL SET-UP}

The experimental part of this investigation was carried out using a conventional shock tube in horizontal orientation, which has a total length of $3.75 \mathrm{~m}$ and a square inner cross section of $80 \mathrm{~mm}$ by $80 \mathrm{~mm}$ [27]. At the shock tube end-wall, a specific wedge test section was designed, manufactured and installed. It has a half apex angle, $\theta_{0}$, of 15 degrees, and accommodates a three-fluid three-zone system: a test cell of a heavy gas ( $\mathrm{SF}_{6}$ ) enclosed by light gas (air) on each side as shown in Fig.1. The shock tube is initially at the atmospheric pressure. The initial expected densities are $\rho_{\text {air }}=1.204 \mathrm{~kg} / \mathrm{m}^{3}$, and $\rho_{S F 6}=6.073 \mathrm{~kg} / \mathrm{m}^{3}$. The corresponding adiabatic exponents are $\gamma_{a i r}=1.4$, and $\gamma_{S F 6}=1.09$. The first air $/ \mathrm{SF}_{6}$ interface forms the gas lens which converts a planar shock wave into a cylindrical one. The same configuration (air/SF 6 fast-slow case) successfully validated in a previous work [24] has been kept: the incident planar shock wave propagates with a Mach number of 1.15 in air, and refracts through an elliptic interface which polar equation writes as:

$$
r(\theta)=r(0) \frac{1-e}{1-e \cos (\theta)}
$$

where $e=W_{t} / W_{i}$ represents the ratio of the transmitted and incident shock wave velocities, and $r(0)=0.159 \mathrm{~m}$ is the location of the interface from the apex. The materialization of the gas lens interface was obtained by a 0.5 - $\mu$ m-thick double layer of nitrocellulose membranes combined with a grid. This grid is obtained by stereo-lithography [REFS]. The second interface $\left(\mathrm{SF}_{6} /\right.$ air $)$, located at $100 \mathrm{~mm}$ from the apex, presents a single mode perturbation with the following polar equation:

$$
r(\theta)=0.1-0.0015\left(1-\cos \left(\frac{2 \pi \theta}{\theta_{0}}\right)\right)
$$

with $\theta_{0}=\arctan \left(\frac{0.04}{0.15}\right)$. This second interface is materialized as the first one. The first and second interfaces are hold by a $8 \times 9.5 \mathrm{~mm}^{2}$ mesh elliptic grid, and $9.5 \times 9.5 \mathrm{~mm}^{2}$ mesh sinusoidal grid, respectively. For recording the evolving flow pattern in the convergent test section, a Z-type Schlieren system is coupled with a Photron Fastcam SA1 high speed digital camera. In addition to the optical diagnostic, pressure transducers recorded pressure histories during each experiment : four of them are located in the corner of the experimental chamber as shown in Fig.1. In the following, we will discuss results obtained from the Schlieren pictures (Fig. 2). The shock wave moves from left to right, and propagates through the air $/ \mathrm{SF}_{6} /$ air three zones. At $t=-10 \mu \mathrm{s}$, the planar shock wave is clearly visible on the left side in the vicinity of the gas lens. When the incident shock wave collides with the air/SF 6 interface, it bifurcates into a transmitted converging cylindrical shock wave which moves in the central zone from $t=65 \mu \mathrm{s}$ to $t=365 \mu \mathrm{s}$. At $t=365 \mu \mathrm{s}$, the converging shock wave impacts the sinusoidally perturbed $\mathrm{SF}_{6} /$ air interface, and the subsequent pictures (from $t=440 \mu \mathrm{s}$ to $t=815 \mu \mathrm{s}$ ) show the different stages of the converging RM instability. As the shock wave hits the interfaces, the nitrocellulose membranes are broken into small pieces. These remnants stay in the vicinity of the interfaces, and are seen as a foamy opaque zone in the Schlieren pictures. At the second interface, the converging shock refracts from the heavy fluid $\left(\mathrm{SF}_{6}\right)$ to the light one (air). Thus, a phase inversion of the perturbation at the interface occurs. We can also note the geometrical distortion of the shock during its refraction at $t=440 \mu \mathrm{s}$. It later stabilizes and recovers its cylindrical shape. The horizontal waves behind the moving interface are the consequence of the support grid. Reaching the apex approximatively at $t=590 \mu$ s, the converging shock wave is reflected back, and expands through the evolving $\mathrm{SF}_{6} /$ air interface at $t=815 \mu \mathrm{s}$.

\section{MONODIMENSIONAL FLOW}

In the following, superscripts ${ }^{(1)}$ and ${ }^{(2)}$ refer to interfaces 1 (the gas lens) and 2 (location of the RM instability), respectively. When waves are considered, subscripts $r$ and $t_{t}$ mean "reflected" and "transmitted". When the geometry is considered, the symbols $r$ and $t$ stand for the distance from the apex of the shock tube, and the time, respectively. The origin of times corresponds to the moment when the planar shock wave hits the interface 1. 


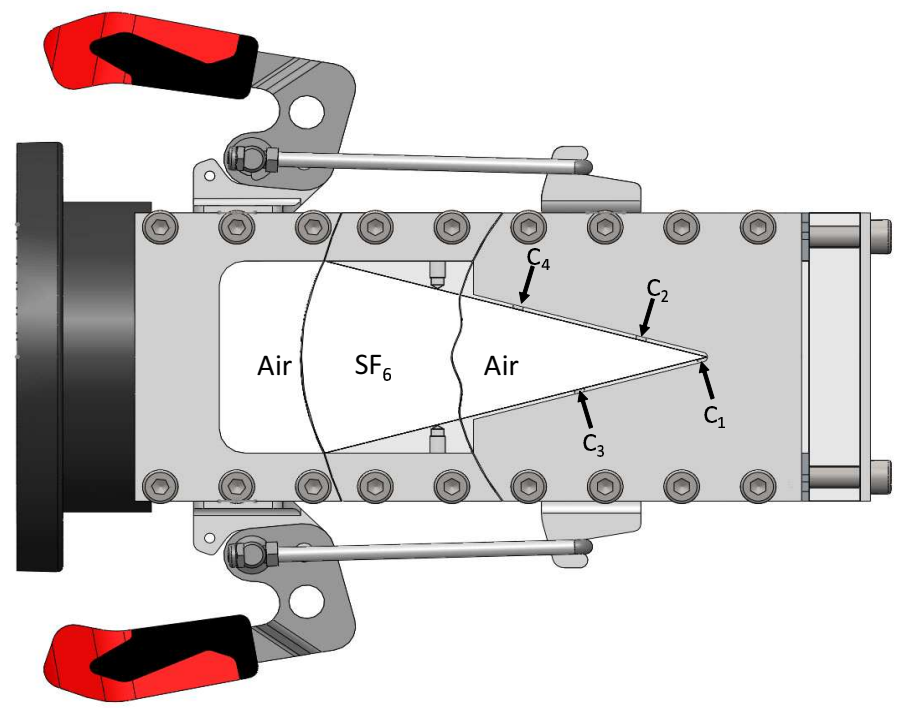

FIG. 1: Scheme of the experimental device adapted on the conventional shock tube, and locations of the pressure gauges.

\section{A. Wave velocities}

In this section, we present the theoretical, and expected wave diagram for our experiments.

Let us recall that the gas lens consists in an air $/ \mathrm{SF}_{6}$ interface. Its purpose is to morph the planar incident shock wave into a cylindrical transmitted one. The Mach number of the incident shock wave, $M_{i}^{(1)}$, is equal to 1.15 in air. The theoretical initial velocity of the transmitted shock wave in $\mathrm{SF}_{6}$ is equal to $W_{t}^{(1)}=165.5 \mathrm{~m} / \mathrm{s}\left(M_{t}^{(1)}=1.226\right)$. The cylindrical shock wave is plainly formed at $r=0.1552 \mathrm{~m}$. In order to predict the trajectory, and the strengthening of the imploding shock wave, the Whitham's approximate geometrical theory [28] is used. This theory gives the Eq. (3) between the Mach number of the converging shock wave, $M$, and its area, $A$ :

$$
\frac{M d M}{M^{2}-1} \lambda(M)+\frac{d A}{A}=0
$$

where $\lambda(M)=\left(1+\frac{2}{\gamma+1} \frac{1-\mu^{2}}{\mu}\right)\left(1+2 \mu+\frac{1}{M^{2}}\right)$, with $\mu^{2}=\frac{(\gamma-1) M^{2}+2}{2 \gamma M^{2}-\gamma+1}$. As $A$ is a known function of $\mathrm{r}, M(r)$ or $M(t)$ can be computed from Eq. (3). The second interface is located at $r=0.1 \mathrm{~m}$. The Eq. (3) gives a theoretical velocity for the shock wave before its impact on the second interface equal to $W_{t}^{(1)}(r=0.1)=171 \mathrm{~m} / \mathrm{s}\left(M_{t}^{(1)}=1.268\right)$. As the velocity of the shock wave in $\mathrm{SF}_{6}$ goes from $165.5 \mathrm{~m} / \mathrm{s}$ to $171 \mathrm{~m} / \mathrm{s}$ between interfaces 1 and 2 , its acceleration is barely noticeable. Once this shock wave reaches the second interface, a shock wave is transmitted in air, and a rarefaction wave is reflected in $\mathrm{SF}_{6}$. The velocity of the transmitted shock wave is initially equal to $W_{t}^{(2)}(r=0.1)=396 \mathrm{~m} / \mathrm{s}$ $\left(M_{t}^{(2)}=1.154\right)$. The head of the rarefaction wave travels back at a velocity equal to $W_{r}^{(2)}(r=0.1)=-75.86 \mathrm{~m} / \mathrm{s}$.

\section{B. Analysis of the experimental $(r, t)$ diagrams}

In this section, we explain the discrepancies between the expected wave diagram, and the actual experimental results.

The experimental data are obtained from two shots which are labeled \#961 and \#962. The initial conditions of these shots (Mach number and gaz composition) were planned to be identical. As far as the shock transit in $\mathrm{SF}_{6}$ is concerned, the two shots give redundant data. The velocity is quasi-constant, and is equal to $168.8 \mathrm{~m} / \mathrm{s}$ and $166.7 \mathrm{~m} / \mathrm{s}$ for shots \#961 and \#962, respectively (Figs. 3-a and b). These values agree with the theoretical velocity which starts from $165.5 \mathrm{~m} / \mathrm{s}$ at the interface 1 to reach $171 \mathrm{~m} / \mathrm{s}$ at the interface 2 .

A clear disagreement between experiments and theory occurs about the reflected wave at the second interface. The experimental measurements show a reflected shock wave which velocity is equal to $-90.6 \mathrm{~m} / \mathrm{s}$ and $-88 \mathrm{~m} / \mathrm{s}$ for shots \#961 and \#962, respectively. Yet the theory predicts a rarefaction wave. This discrepancy is explained by the grid bars. They generate the reflected shock wave (see Appendix A). The trajectory of this shock wave can be theoretically estimated. It is in reasonable agreement with the experimental data: at $t=0.6 \mathrm{~ms}$, its radius is $122 \mathrm{~mm}$ 

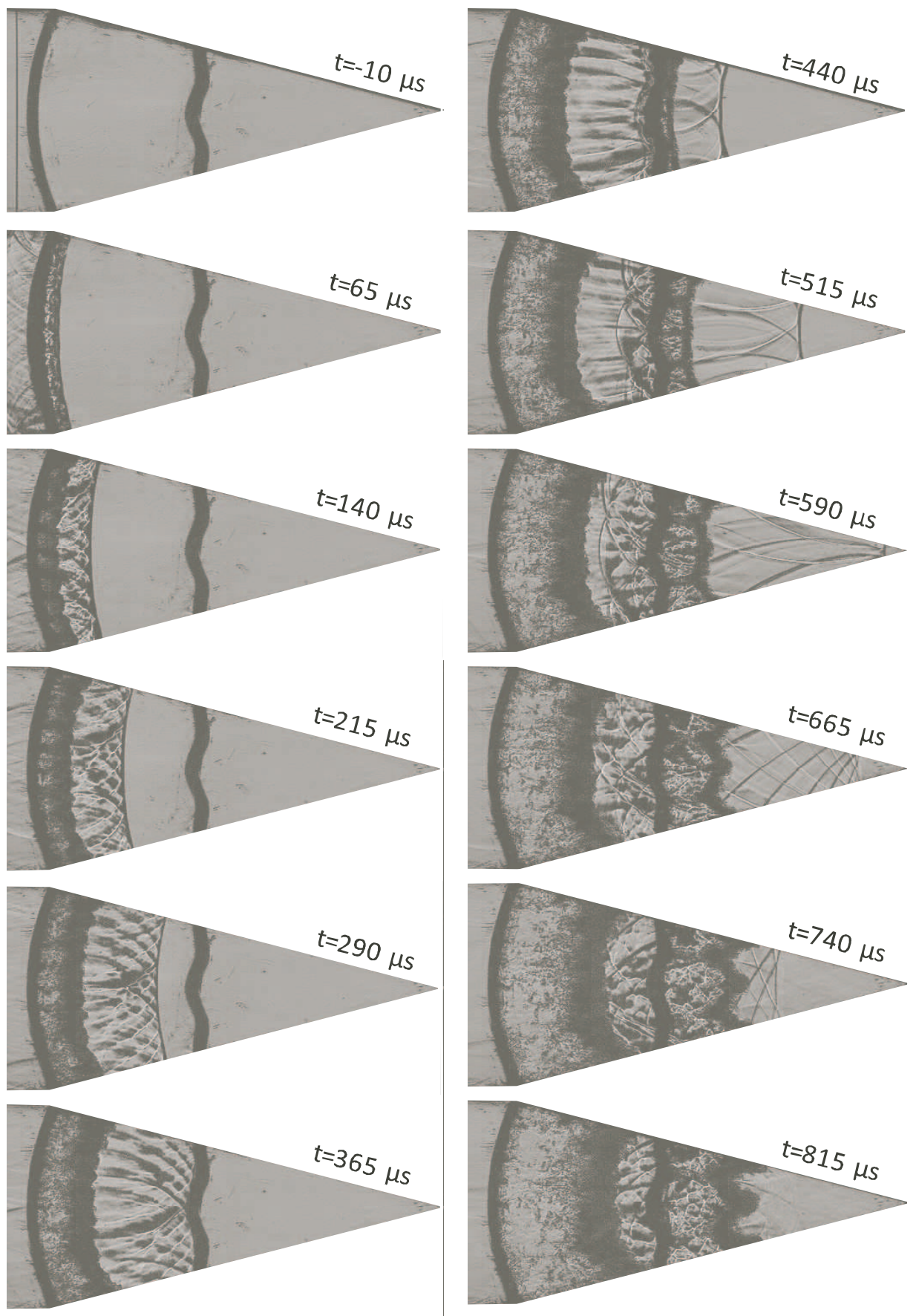

FIG. 2: Sequence of schlieren pictures (from run \#961) showing the evolution of the converging Richtmyer-Meshkov instability at the $\mathrm{SF}_{6} /$ air interface materialized by 2 layers of $0.5-\mu \mathrm{m}$-thick nitrocellulose membrane recovered in sandwich on a grid support $\left(9.5 \times 9.5 \mathrm{~mm}^{2}\right)$.

in the experiment, and the theory predicts $119 \mathrm{~mm}$.

We now focus on the transmitted shock wave in the apex section. The shock wave velocity, $W_{t}^{(2)}$, and the travel time of the shock wave between interface 2 and the apex, $\Delta t_{\text {focus }}$, for the two experimental cases are compared with the theoretical values in Table I. For the shot \#961, the relative discrepancies between experimental and theoretical data are within the error bar of the experiments. Therefore, we will consider that the gases are pure for shot \#961. For the shot \#962, the velocity of the transmitted shock wave is slower than expected. The relative discrepancy with respect to the theoretical velocity is about $16 \%$. In order to explain this value, two reasons can be put forward. 
a)

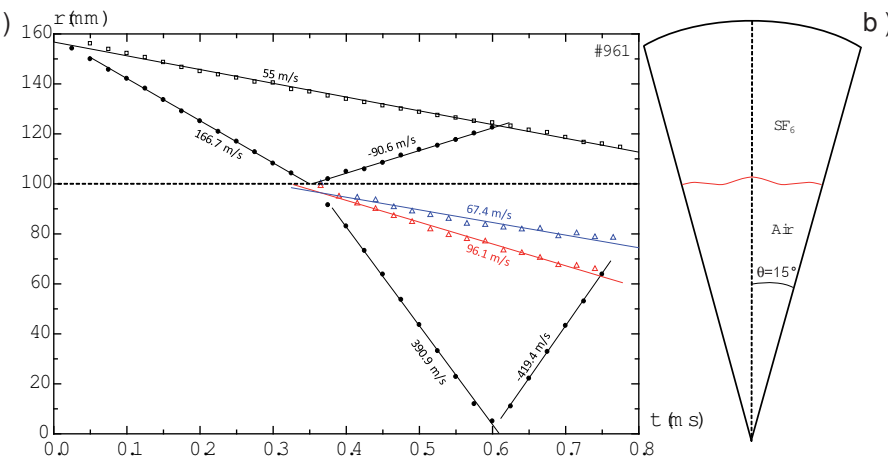

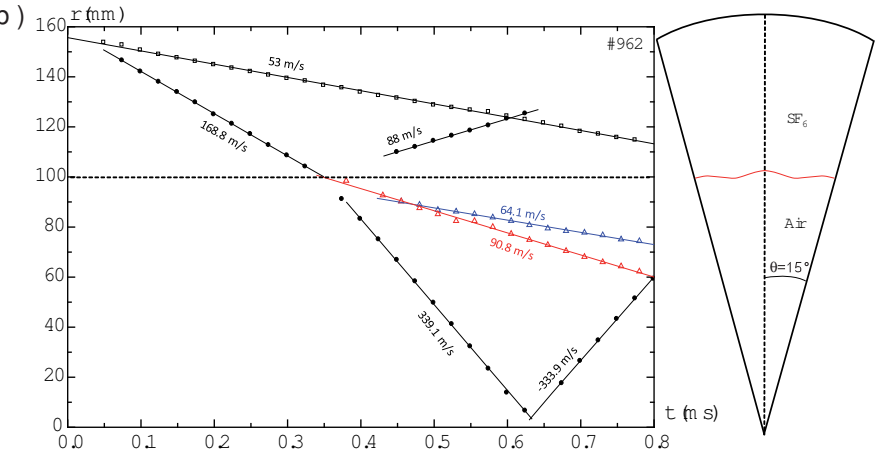

FIG. 3: One-dimensional wave-diagrams (r-t plane) reconstructed from pressure measurements, and Schlieren pictures. a- shot \#961, b- shot \#962.

TABLE I: Velocity, $W_{t}^{(2)}$, of the transmitted shock wave at the interface 2 , and time lapse, $\Delta t_{f o c u s}$, between the shock passage at the interface 2 , and the focusing at the apex.

\begin{tabular}{lcccc}
\hline & Shot \#961 & Shot \#962 & $\begin{array}{c}\text { Theory } \\
\text { (pure gases) }\end{array}$ & $\begin{array}{c}\text { Theory } \\
\text { (polluted air) }\end{array}$ \\
\hline$W_{t}^{(2)}(\mathbf{m} / \mathbf{s})$ & 390.9 & 333.8 & 396.1 & 334. \\
$\Delta t_{\text {focus }}(\mathbf{m s})$ & 0.238 & 0.280 & 0.234 & 0.277 \\
\hline
\end{tabular}

Firstly, the membrane which is initially held by the grid, and which separates the $\mathrm{SF}_{6}$ from air, could absorb a part of the incident shock wave energy. However, if the membrane at the second interface had any influence on the flow, it would have been seen on the shot \#961, and at the interface 1. Therefore, we dismiss this hypothesis. Secondly, the air in the apex section of the shock tube could be polluted by $\mathrm{SF}_{6}$. The resulting mixture would be heavier than air, and the transmitted shock wave slower than expected. A 8.8\% at. $\mathrm{SF}_{6}$ polluted air would give the correct value for $W_{t}^{(2)}$ and $\Delta t_{\text {focus }}$. For the shot \#962, we will now consider that the gas in the apex section of the shock tube is a $\mathrm{SF}_{6}$-air mix. The initial density of this gas is $\rho_{M i x}^{0}=1.633 \mathrm{~kg} / \mathrm{m}^{3}$, its adiabatic coefficient is $\gamma_{M i x}=1.307$, and the initial sound speed is $c_{M i x}=284.78 \mathrm{~m} / \mathrm{s}$.

The post-shock Atwood numbers are $A t^{+}=-0.687$, and $A t^{+}=-0.594$ for shots \#961, and \#962, respectively. As far as the RM instability is concerned, the Atwood number at interface 2 is defined as $A t=\left(\rho_{\text {air }}-\rho_{S F 6}\right) /\left(\rho_{\text {air }}+\rho_{S F 6}\right)$. Should the Rayleigh-Taylor instability be concerned, the Atwood number would be the magnitude of the previous definition.

Figures 4 and 5 present the theoretical, and experimental wave $(r, t)$ diagrams for the two shots.

In order to further assess our previous conclusions about the composition of the gases, numerical simulations have been performed with the multimaterial Arbitrary Lagrangian Eulerian (ALE) package of the Hesione code [29, 30]. The subsequent wave trajectories are also presented in Figs. 4 and 5. They match the experiments. A further result which is deduced from thes good agreements is that the membranes have no influence on the wave dynamics.

\section{SHAPE OF THE PERTURBATION AT THE SECOND INTERFACE}

\section{A. Grid imprint}

In this section, we focus on the shape of the distorted interface. Pure air and $\mathrm{SF}_{6}$ gases are considered unless otherwise specified. The bars on the gas lens (interface 1) are not considered since they do not influence the circular converging shock wave in $\mathrm{SF}_{6}$.

As seen on the experimental pictures of Fig. 2, the shape of the perturbed interface develops a sawtooth shape. This 


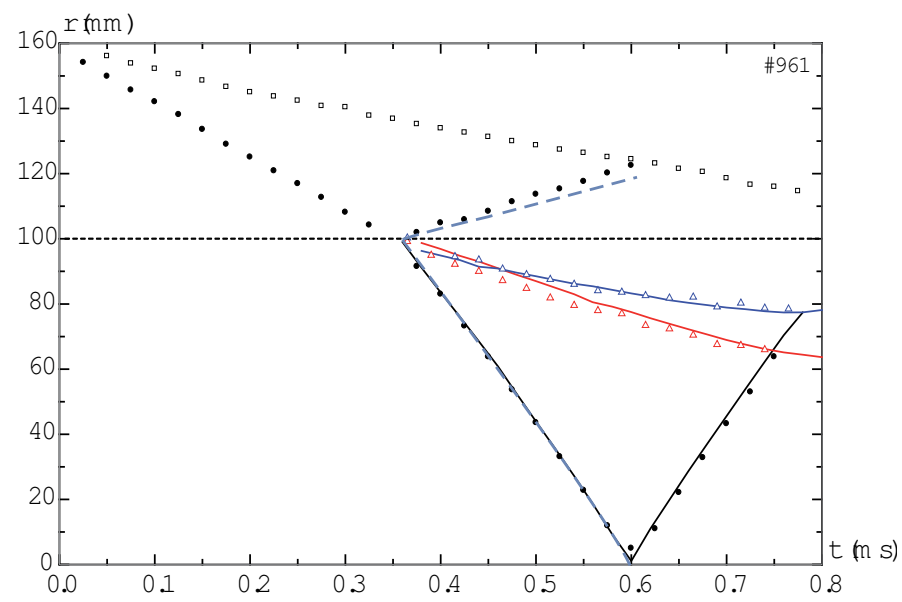

FIG. 4: (r,t) diagram for shot \#961. Symbols represent experimental data. Full and dashed lines are from simulation and theoretical results, respectively.

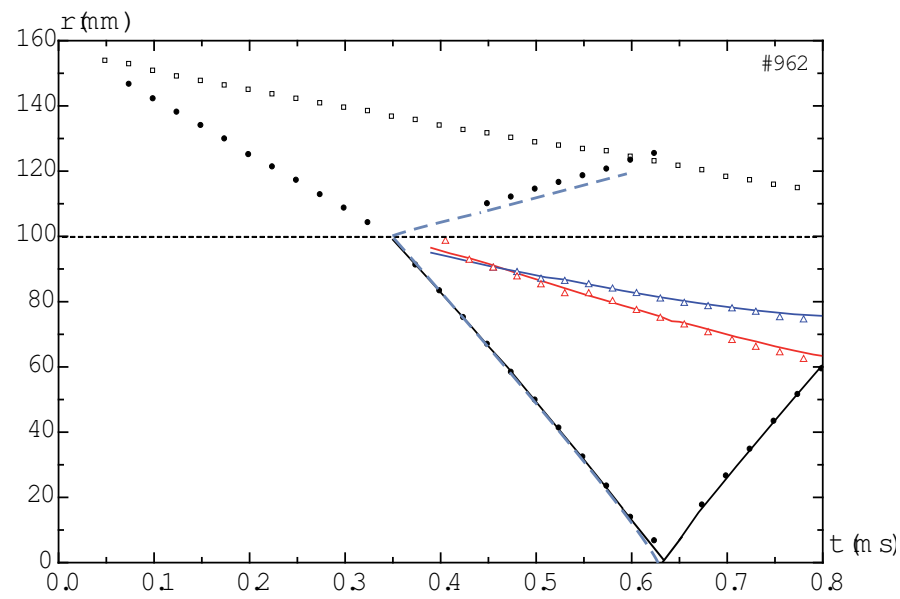

FIG. 5: (r,t) diagram for shot \#962. Symbols represent experimental data. Full and dashed lines are from simulation and theoretical results, respectively.

is not what is expected from the growth of a single mode sinusoidal initial perturbation. The grid bars are to be taken into account to explain this peculiar shape of the interface. This inference is established with numerical simulations. In order to compute the grid bars (motionless areas), the pure Eulerian package of the Hesione must be used. This package requires a cartesian mesh, which implies that the tilted walls of the convergent shock tube are modeled as staircases. This artificial roughness of the walls generates a numerical boundary layer. However, if the mesh is fine enough, the effect of this boundary layer is negligible: the tip of the jet is slightly modified, but its amplitude remains unaffected. This can be seen in Fig. 6 (upper part) which presents an Eulerian computation of the shock tube without grid.

In order to see if the grid bars modify the growth of the perturbation at the interface 2, eulerian simulations with these bars have been performed (bottom part of Fig. 6). The cell sizes of the mesh are $\Delta x=\Delta y=1.2510^{-4} \mathrm{~m}$. This corresponds to 205 points per perturbation wavelength (ppw). Only half of the shock tube is considered here.

Without any bar, the interface displays the common features of a single mode sinusoidal RM instability in the nonlinear regime, i.e. a rounded bubble, and a long jet with a mushroom cap. When the grid is taken into account, the shape of the interface becomes sawtooth like. Furthermore, additional small jets are generated at the peak and the valley of the interface. There are the consequence of trailing small scale perturbations growing in the wake of the bars. Afterwards, due to the RM instability, these trailing dents on the interface reverse, and turn into small extra jets.

The numerical convergence of the Hesione code has been studied through a zoning study. A new grid resolution 


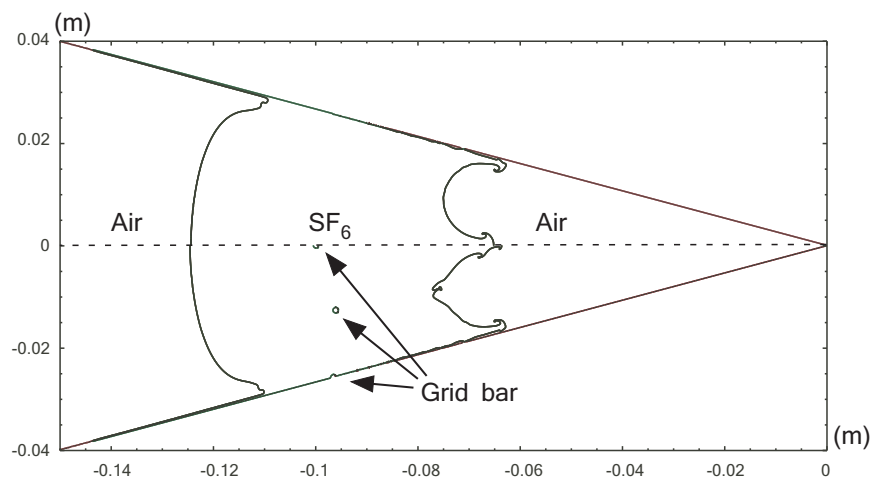

FIG. 6: Numerical simulations. Interface shapes shortly before the re-shock of interface 2. Top: without the grid bars. Bottom: with the grid bars.

has been considered: $\Delta x=\Delta y=8.3310^{-5} \mathrm{~m}$ (307 ppw). Furthermore, the full width of the shock tube has been computed in order to suppress any imposed symmetry on the $x$-axis. As shown in Fig. 7 , the 205 and 307 ppw interfaces superimpose, with the exception of the aditional small jets which are slightly different.

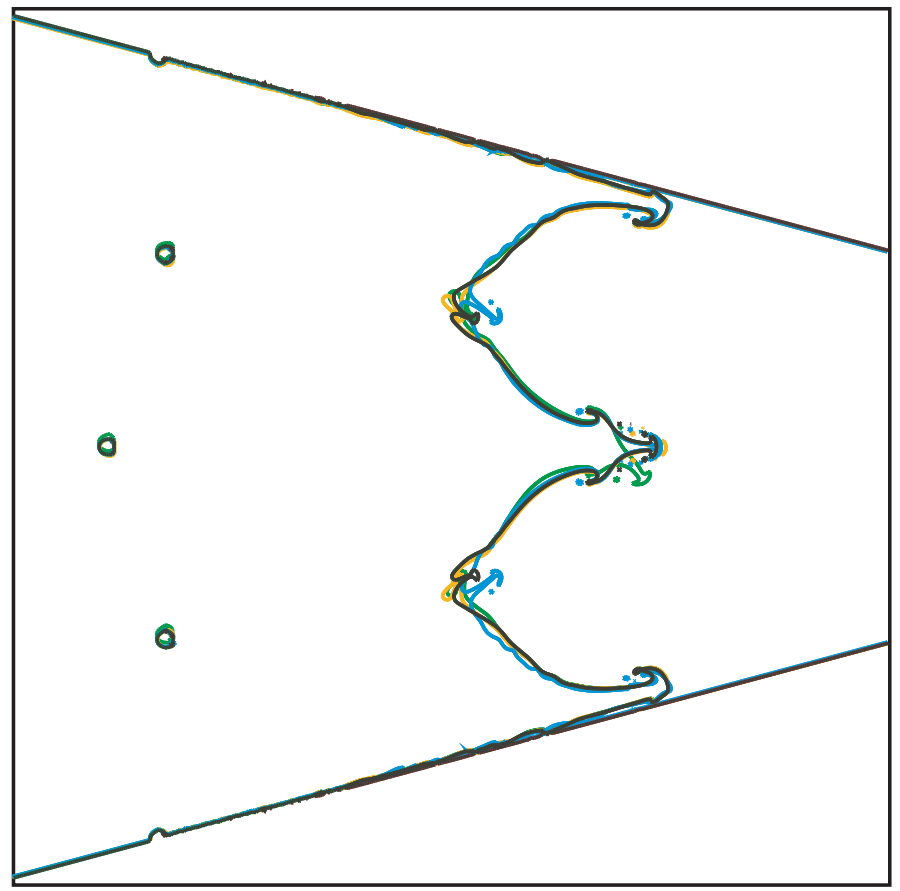

FIG. 7: Numerical simulations. Interface shapes shortly before the interface 2 re-shock time. Black, blue, orange, and green shapes are results from numerical simulation with 205, 206, $307 \mathrm{ppw}$, and $205 \mathrm{ppw}$ with a $\Delta y=+0.3 \mathrm{~mm}$ shifted interface, respectively.

In order to check the sensitivity of the flow symmetry to the parity of the number of points, we run a $206 \mathrm{ppw}$ simulation. The results are the same as those obtained from the $205 \mathrm{ppw}$ computation. As a result, we consider that the $205 \mathrm{ppw}$ resolution is fine enough to describe correctly our shock tube. Let us note that the symmetry of the flow with respect to the $x$-axis remains in these simulations which compute the whole tube. In experiments, it could happen that the stereo-lithographed grid is slightly out of alignement with respect to the plan of symmetry. In order to illustrate the sensitivity to such a shifting, we have run a simulation with a $\Delta y=+0.3 \mathrm{~mm}$ shifted interface. This shifting does not change the general shape of the interface. However, the additional small jets are modified, and the 
one near the $x$-axis now grows downwards (Fig. 7). This result shows that the directions, and the amplitudes of the small scale features of the interface are highly sensitive to any offset of the grid.

\section{B. Analysis of the experimental pictures}

The Schlieren technique reveals the density gradients in the flow. Our intention is to track the interface through this means. However, the experimental pictures display a lot of features in addition to the interface signal. In this section, by comparing experimental results with numerical simulations, we detail the different causes for the Schlieren signals, and identify the actual location of the interface.

For the following numerical results, we consider that the initial position of the interface is ideal (no offset). Figure 8 presents the result of the Hesione simulation at a time just before the re-shock: the pressure field is superimposed on the contours of the interfaces, and the bars for shot \#961. This simulation shows that Von Karman alleys appear downstream the bars. Accoustic and shock waves are also displayed.

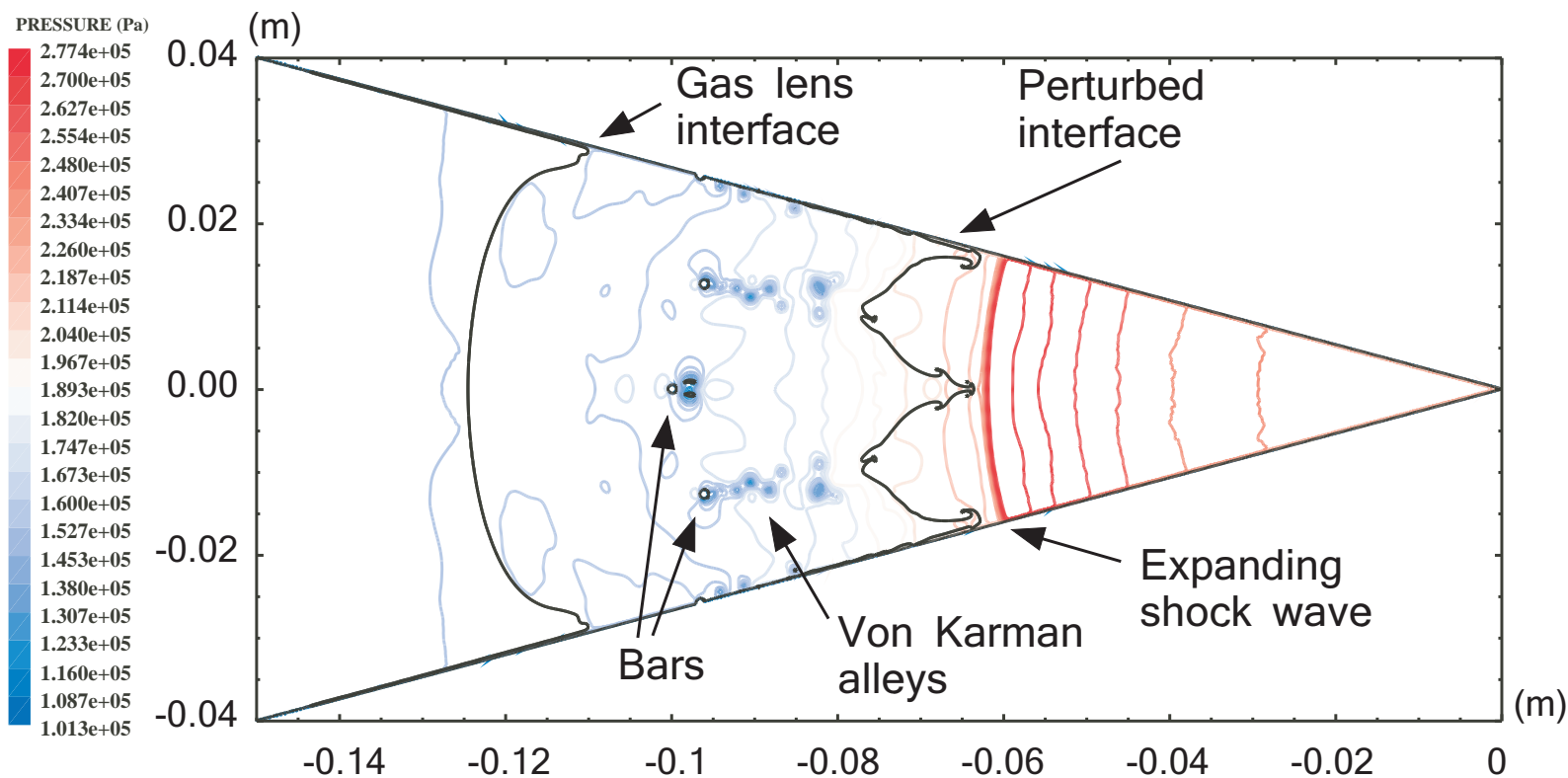

FIG. 8: Numerical simulation of shot \#961. Interface shape and pressure field shortly before the re-shock of interface 2 $(\mathrm{t}=0.74 \mathrm{~ms})$.

Density gradients can be generated by shock waves, accoustic waves, Von Karman alleys, and interfaces between different materials. Flow momentum can also generate density gradients. In our case, this occurs in the jet vicinity where matter gathers. Figure 9 presents the density maps in the jet area at $t=0.53 \mathrm{~ms}$ and $\mathrm{t}=0.74 \mathrm{~ms}$.

As indicated by arrows in Fig. 9, dense areas can be spotted at the base of the jet. We have simulated the Schlieren diagnostic of the Hesione computation. For the shot \#961, results are presented in Figs. 10 and 11 at $\mathrm{t}=0.53 \mathrm{~ms}$ and $\mathrm{t}=0.74 \mathrm{~ms}$, respectively. They are to be compared with the experimental pictures (Figs. 10-a, and 11a). In the real shock tube, the membrane remnants stop the light propagation and appear in the pictures. However, they are not taken into account in the numerical simulations. 

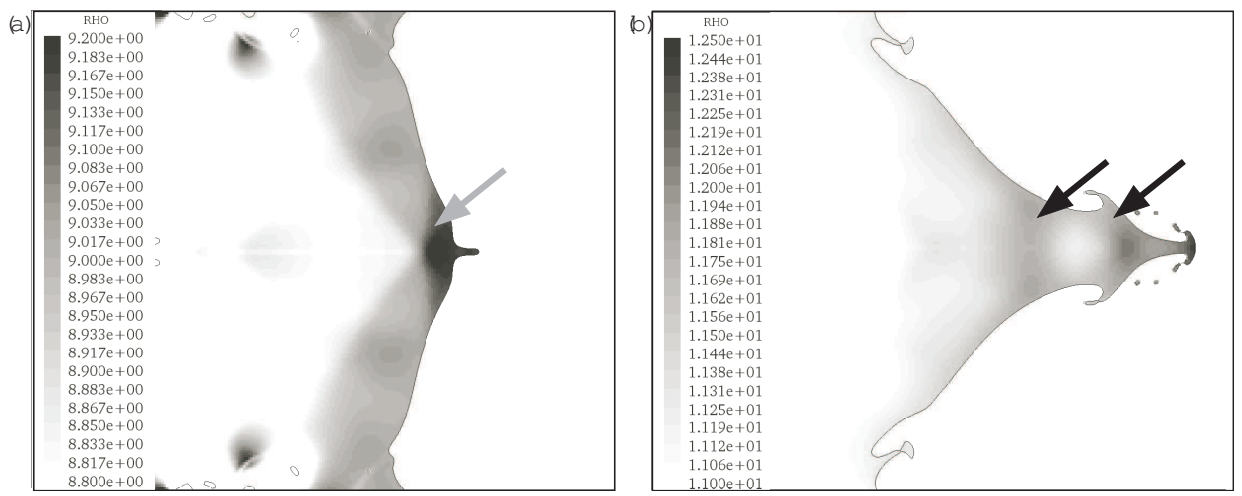

FIG. 9: Numerical simulation of shot \#961. Density map near the jet at interface 2 . a- $t=0.53 \mathrm{~ms} . \mathrm{b}-\mathrm{t}=0.74 \mathrm{~ms}$
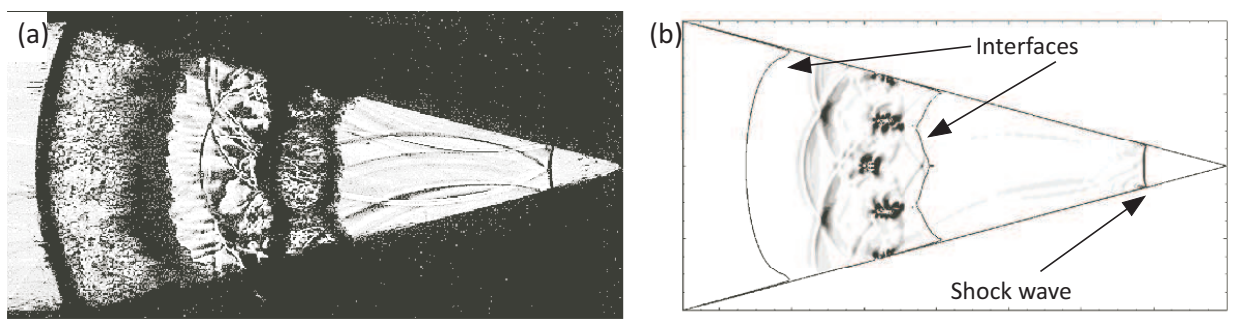

(c)

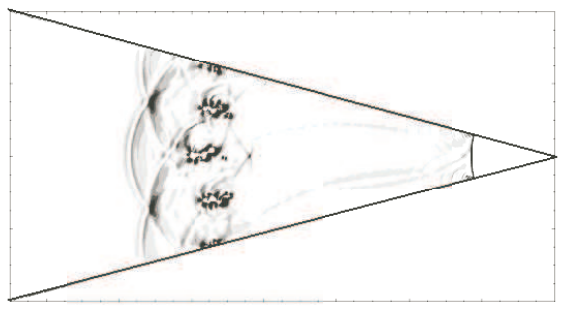

(d)

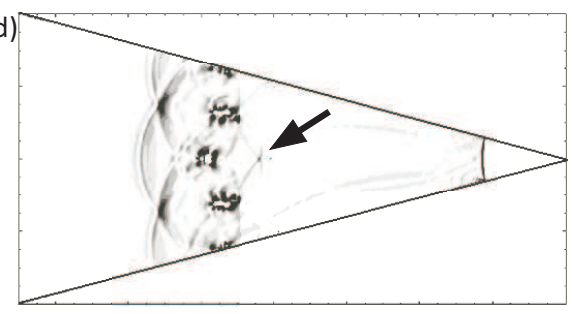

FIG. 10: Shot \#961 at t=0.53 ms. a- Experimental picture. b- Numerical Schlieren picture and superimposed interfaces (no grid offset). c- Numerical Schlieren picture (grid offset: $\Delta y=+0.3 \mathrm{~mm}$ ). d- Numerical Schlieren picture (no grid offset).

In Figs. 10 and 11 (b,c and d parts), the diagnostic simulations are displayed. The shock waves, the accoustic waves, and the Von Karman alleys can be identified. The dense areas at the base of the jet are also tracked by the Schlieren diagnostic (see arrows in the Figs. 10-d, and 11-d). On the Figs. 10-b, and 11-b, the numerical interfaces are purposely added to see how, in the vicinity of the jet, it becames difficult to separate the interface signal from the dense area signal.

Let us now compare the numerical $\mathrm{SF}_{6} /$ air interface with the corresponding experimental photograph for shot \#961 (Fig. 12).

We emphasize that both numerical and experimental data are synchronized without any time shifting. At this moment, the numerical and experimental shock waves superimpose (Figs. 12-a and b). On the photograph (Fig. 12-a), the interface between $\mathrm{SF}_{6}$ and air is blurred by the membrane remnants. Figure 12-c shows that the computed interface overlaps the left boundary of the remnant zone. In earlier works [31], we showed that when a shock wave goes from an heavy gas to a light one, the membrane remmants are expelled ahead of the interface. In this heavy-to-light configuration, we also showed they have no influence on the interface dynamics. The Fig. 12-c confirms our previous assertions: the foamy zone consists of membrane remnants, stays ahead the $\mathrm{SF}_{6}$ /air interface, and does not reduce the perturbation growth. Using the numerical results (Figs. 8 and 11-c or d), we can also infer that the shimmering aspect of the space between the grid bars and the interface is mainly due to the Van Karman alleys and accoustic waves. The dense areas at the base of the jet are also identified on the experimental pictures. These results are confirmed by shot \#962 (Fig. 13). 

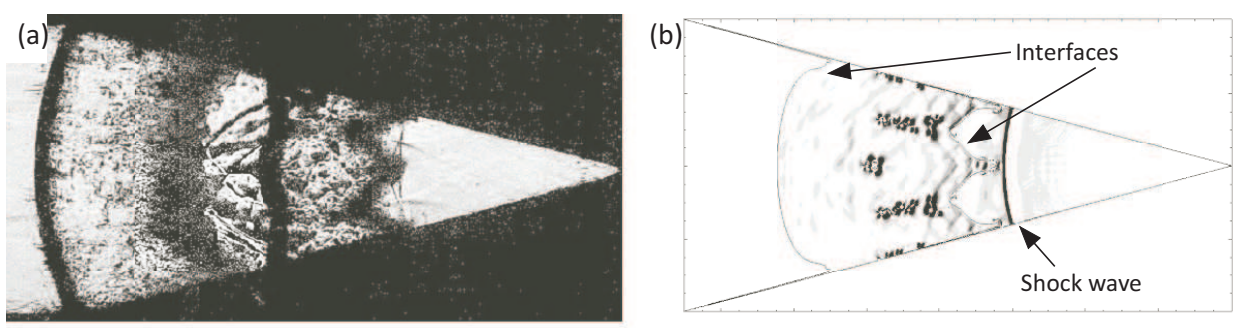

(c)
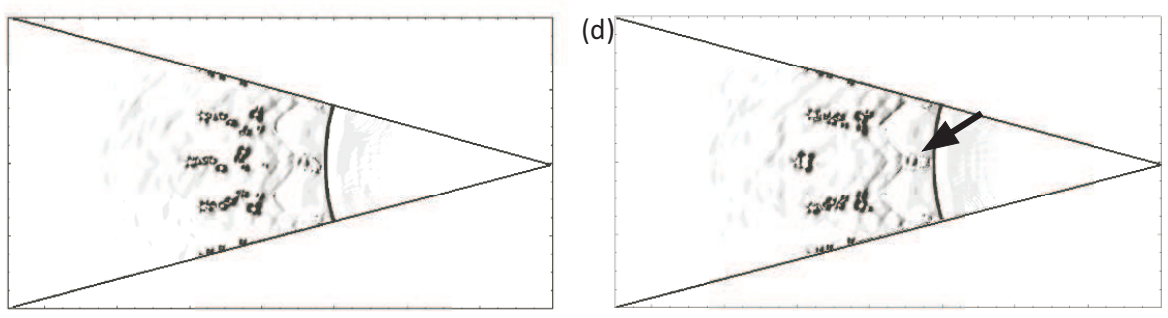

FIG. 11: Shot \#961 at t=0.74 ms. a- Experimental picture. b- Numerical Schlieren picture and superimposed interfaces (no grid offset). c- Numerical Schlieren picture (grid offset: $\Delta y=+0.3 \mathrm{~mm}$ ). d- Numerical Schlieren picture (no grid offset).

a)

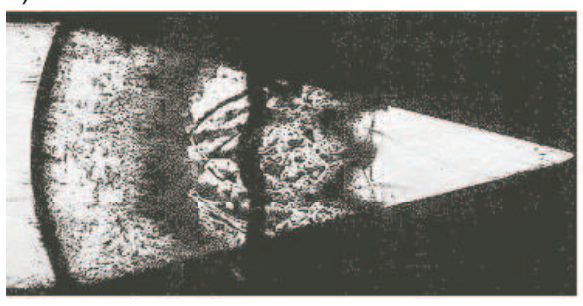

b) $0.04(\mathrm{~m})$

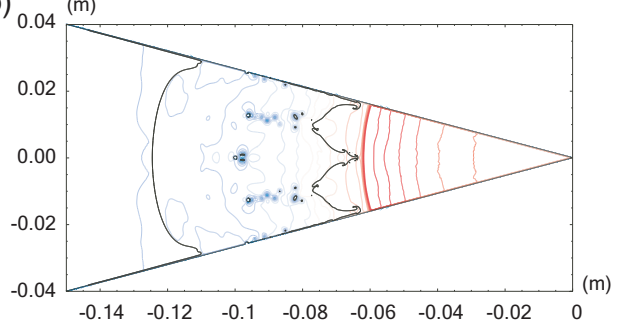

c)

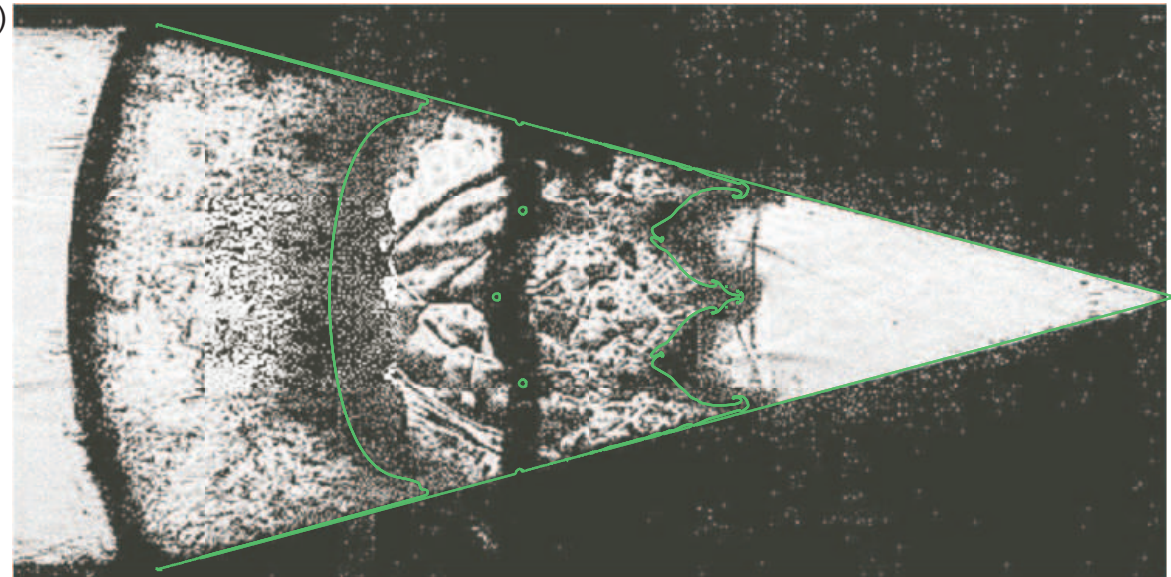

FIG. 12: Shot \#961 before the re-shock of interface $2(\mathrm{t}=0.74 \mathrm{~ms})$. a- Experimental picture. b- Numerical simulation with the interfaces (black lines), and the pressure field (colored contours). c- Superposition of the experimental image, and the numerical interfaces (green).

First conclusions can be drawn from these comparisons:

- the grid bars influence the growth of the perturbation at the interface,

- the resulting shape of the interface is sawtooth like,

- if the grid bars are taken into account, the agreement between numerical simulations and experimental data is good, 
a)

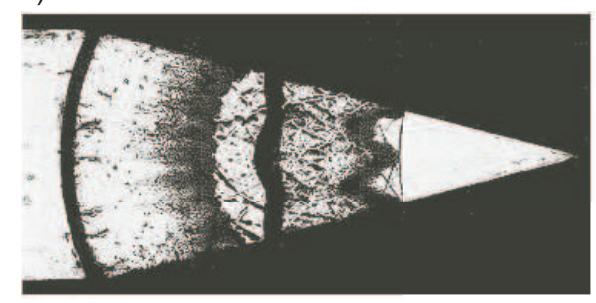

b) $0.04(\mathrm{~m})$

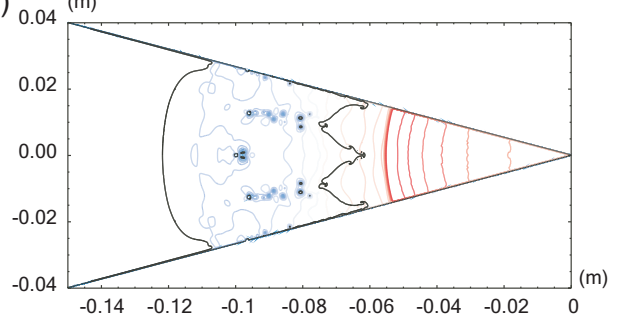

c)

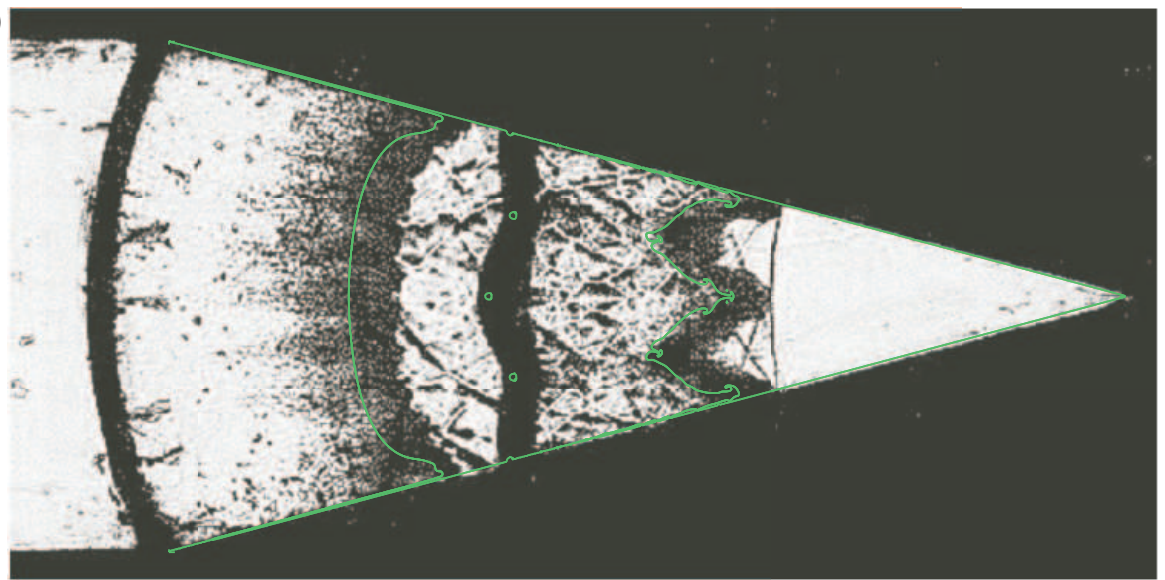

FIG. 13: Shot \#962 before the re-shock of interface $2(\mathrm{t}=0.78 \mathrm{~ms})$. a- Experimental picture. b- Numerical simulation with the interfaces (black lines), and the pressure field (colored contours). c- Superposition of the experimental image and the numerical interfaces (green).

- it is confirmed that in the heavy-to-light configuration, the membrane remnants are expelled away from the interface, and do not influence its dynamics,

- dense areas at the base of the jet are seen by the Schlieren diagnostic.

\section{GROWTH OF THE INSTABILITY}

In this section, we show that, even if the grid bars induce a change of the interface shape, the growth of the fundamental mode is not so modified. We also characterize the regimes of the instability.

\section{A. Amplitude of the perturbation}

In the following, as we focus on the interface dynamics, the origin of time is now taken at the moment when the interface 2 is set into motion. We will denote the time as $\tau(\tau=t-0.36 \mathrm{~ms})$.

From the previous section, we can interpret the experimental interface area as depicted by Fig. 14-a. Let us denote the locations of the peak and valley of the real interface, as points $A$ and $B$, and the locations of the peak and valley of the right side limit of the foamy zone, as points $C$ and $D$.

The key data to estimate is the amplitude of the real interface which writes as $a(t)=\frac{1}{2}\left(r_{A}-r_{B}\right)$. Due to the dense areas at the base of the jets, the location, $r_{B}$, cannot be precisely estimated in the experiments. However, the trajectories of the other points $A, C$ and $D$ can be measured (Fig. 14-b, and Fig. 15-b). Let us note that the width, $\delta$, of the membrane remnant zone grows steadily with time (Figs. 14-c and 15-c). As a result, we make the following assumption in order to estimate the location of point $B$ :

$$
r_{B} \approx r_{D}-\delta
$$


a)
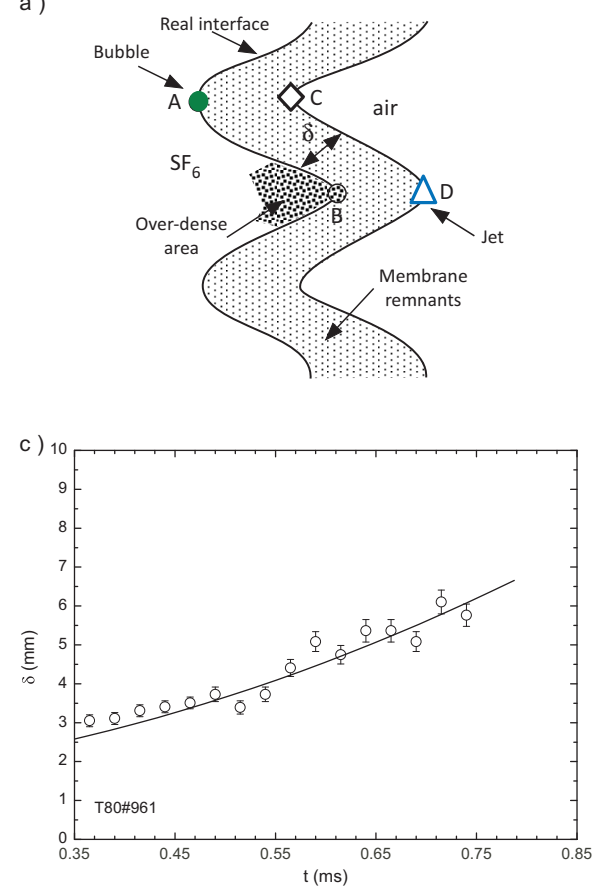

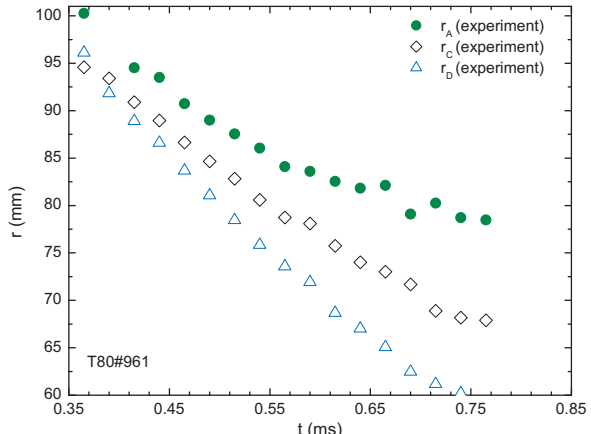

d)

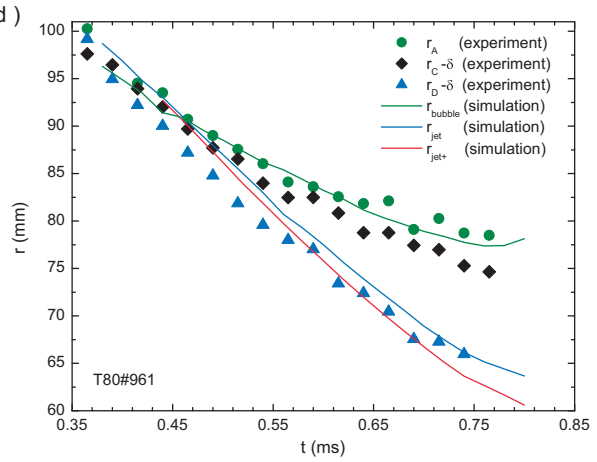

FIG. 14: Interface study for the shot \#961. a- Schematic of the Schlieren picture, b- experimental location for points $A, C, D$, c- growth of the foamy zone, d- comparison between numerical and experimental estimates of the locations of the interface peak and valley. Symbols are obtained from the experiments and formula (4). Curves are from the numerical simulations. For the trajectory of the jet, the two curves are obtained by taking into account or not the extra small jet which is induced by the grid.

The Figures 14-d and 15-d present the trajectories of the peak and valley of the interface for the two shots where the experimental location of the jet, $r_{B}$, is computed with formula (4). They are compared with two numerical results. The first one is obtained by taking into account the amplitude of the small jet which appears at the tip of the main jet, and the second one by removing this amplitude. The agreement between numerical and experimental trajectories is good. This good agreement shows that our understanding of the experimental phenomenology which has been established in the previous section is valid through time. The amplitude of the perturbation at the interface is displayed on Fig. 16 for the two shots.

\section{B. Regime of the instability}

In the planar geometry, the linear phase of the RM and the RT instabilities is defined by $|k a(\tau)|<1$, where $k$ is the wavenumber. For the RM instability, this phase is characterized by a perturbation growth which linearly increases over time. In the nonlinear regime, the instability then saturates to a logarithmic growth [32]. In the present study, the growth of the perturbation seems to linearly depends on time (Fig. 16) with no saturation.

In the cylindrical geometry, $k a(t)$ is replaced by $n a(t) / R$ where $n$ is the mode number of the perturbation, and $R$ the radius of the unperturbed interface. At first order, the velocity of the interface can be considered as constant and equal to $\Delta U$. As a result, $R$ can be approximated by $R(t)=0.1-\Delta U t$, with $\Delta U=-82.4 \mathrm{~m} / \mathrm{s}$ and $-79 \mathrm{~m} / \mathrm{s}$ for shots \#961 and \#962. Just before the re-shock, $|n a / R| \approx 1.52$ for the shot \#961. We conclude that, despite the linear growth of the perturbation, the instability should be in the nonlinear regime at the re-shock time. It must be checked that this lack of saturation is a general feature of the nonlinear growth in the convergent geometry, and not a side effect due to the grid bars. 
a )
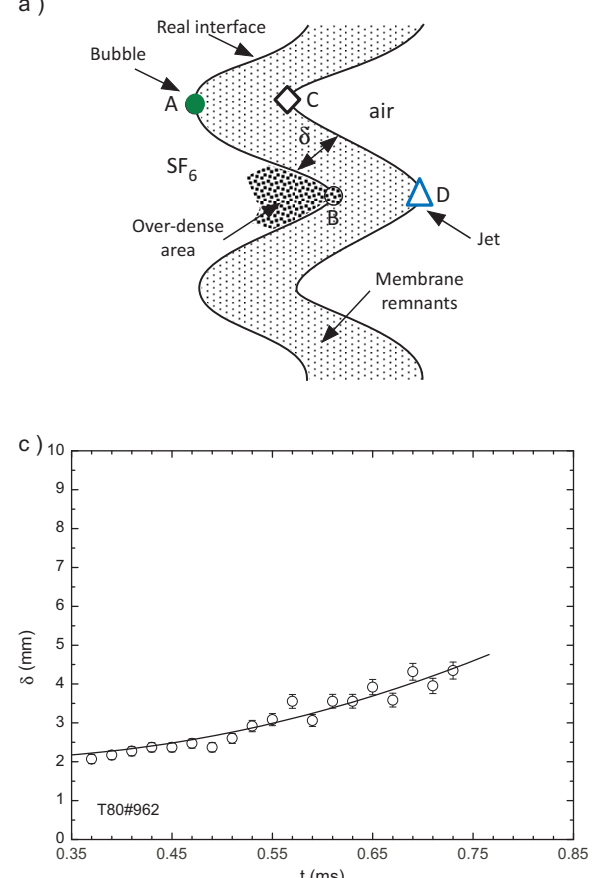

b)

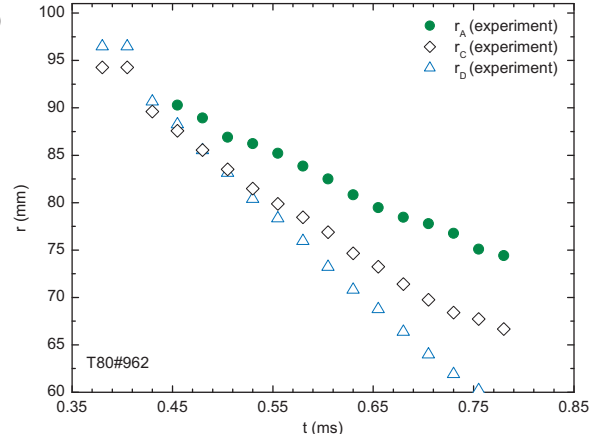

d)

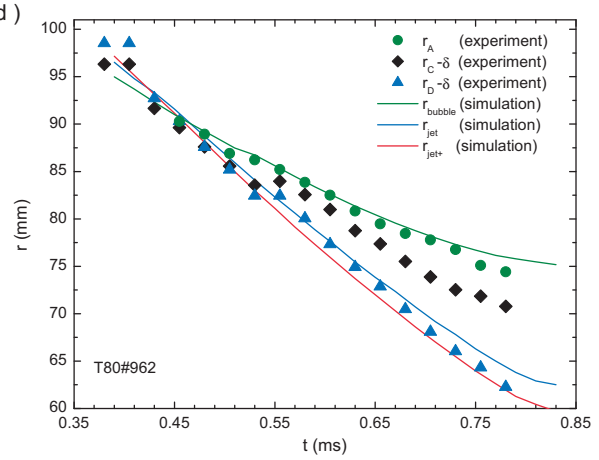

FIG. 15: Interface study for the shot \#962. a- Schematic of the Schlieren picture, b- experimental location for points $A, C, D$, c- growth of the foamy zone, d- comparison between numerical and experimental estimates of the locations of the interface peak and valley. Symbols are obtained from the experiments and formula (4). Curves are from the numerical simulations. For the trajectory of the jet, the two curves are obtained by taking into account or not the extra small jet which is induced by the grid
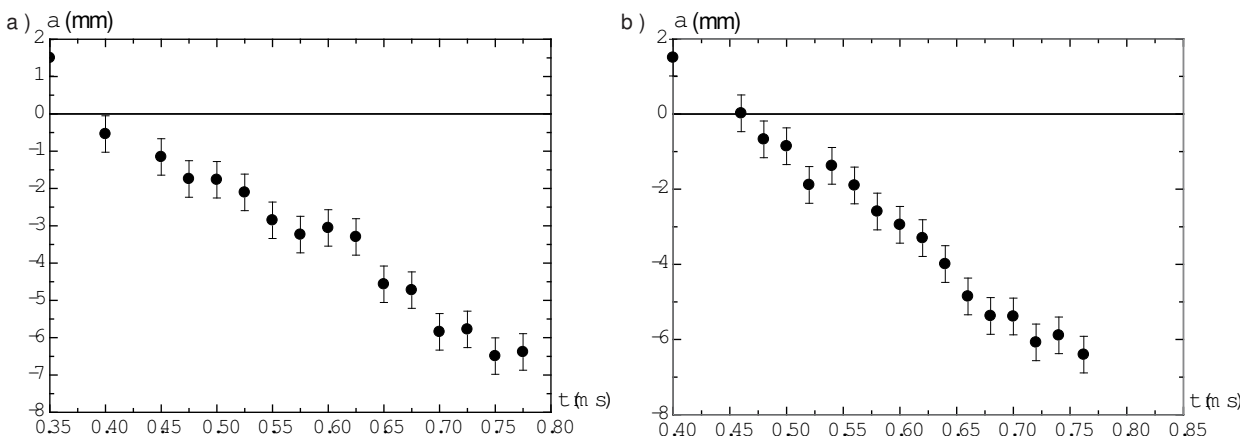

FIG. 16: Amplitude of the perturbation. a- shot \#961. b- shot \#962.

\section{Influence of the grid}

As seen before, the grid induces a sawtooth shape at the interface. In the following, Fourier analyses of the shape of the interface are performed in order to study the influence of the grid bars in the spectral domain. The Figure 17-a presents for the shot \#961, the growth of the first three harmonics for simulations with and without the grid bars.

It appears that the growth of the first harmonics with the grid bars is similar to the one without the grid. However, a $14 \%$ increase of the growth rate can be noticed due to the grid. This value is constant over time. The influence of the grid appears to be more important on the amplitude, $\eta_{2}$ of the second harmonics. The latter should be negative since the weakly nonlinear theory [33] predicts $\eta_{2}=\frac{\nu_{0}^{2}(2 A n-1) \tau^{2}}{4 r_{0}^{2}}$ with $A=-0.687$ and $\nu_{0}=\left.\frac{d a}{d t}\right|_{\tau=0}$. The numerical simulation with the grid shows a positive growth of the second harmonics. However, around $\tau=0.15 \mathrm{~ms}$, the growth of the second harmonics returns to a classical negative growth, with a smaller growth rate than expected. The growth of the third harmonics is, as the first one, enhanced by the grid bars. Even if substantial discrepancies exist on 
a)

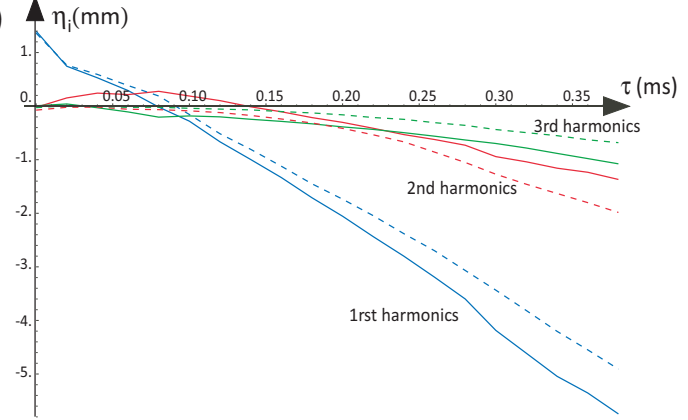

b)

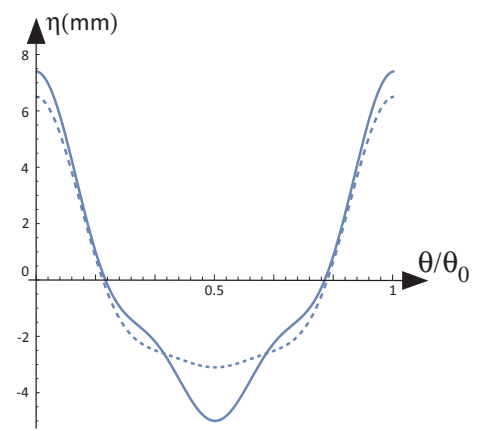

FIG. 17: Analysis of the interface shape for the shot \#961. a- Growth of the first three harmonics. b- Reconstructed shape at $\tau=0.35 \mathrm{~ms}$. Full and dotted curves are obtained from simulations with and without the grid bars, respectively.

the growth rates of the second and third harmonics, the amplitudes of these harmonics remain small in comparison with the amplitude of the first harmonics. As a result, the feed-back on the first harmonics stays low: the influence of the grid on the first harmonics remains limited. These conclusions are in accordance with the ones which were drawn from a previous study [34] in the planar case: the grid adds short-wavelength (swl) perturbations to the singlemode interface with low feed-back on the fundamental mode. Another way to check that our experiment is a good approximation of the canonical single mode problem is to reconstruct the shape of the interface with the first three modes. The Figure 17-b presents such a reconstruction at $\tau=0.35 \mathrm{~ms}$. Even if the fundamental mode amplitudes are similar with and without the influence of the grid, the small discrepancies between the lower modes are enough to transform a round shape in a sawtooth one. To conclude, these comparisons show that the lack of logarithmic saturation of the growth of the perturbation is not due to the grid.

\section{Theoretical modeling}

All theoretical models about the growth of the RM and RT instability in the cylindrical geometry deal with the canonical single mode problem. It is important to estimate the accuracy of such models either by comparison with experiments or simulations. If a $14 \%$ discrepancy on the growth rate is tolerated, our experimental results could be directly used as a reference. However, to reduce the uncertainties and specifically work on the fundamental mode, the numerical simulation which has been performed without the grid will be used. In order to estimate if our case is a pure RM instability (no post-shock acceleration of the interface), a numerical simulation with no perturbation at the $\mathrm{SF}_{6}$ /air interface has been performed. It appears that the 1D motion of the interface undergoes a slight deceleration as it travels towards the apex (Fig. 18). This means that the instability is a RT instability which is initiated by a RM instability.

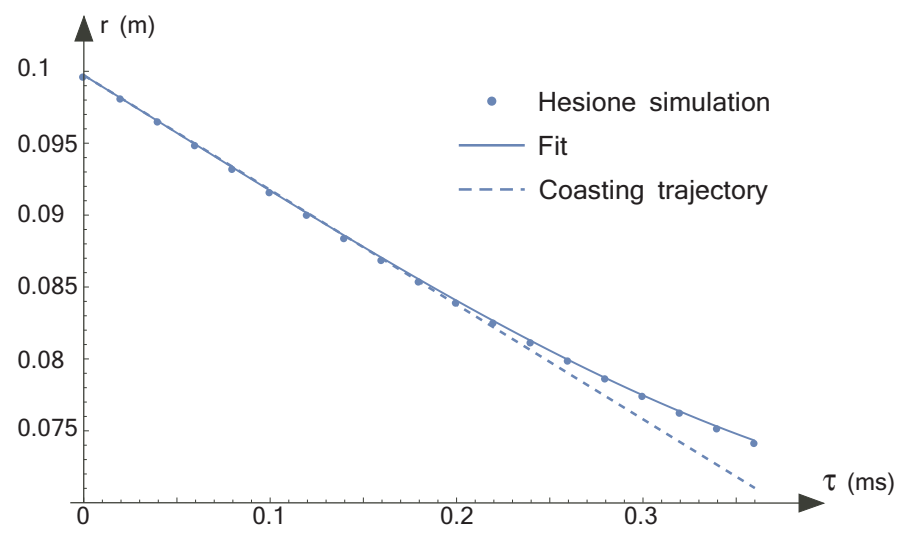

FIG. 18: Trajectory of the unperturbed interface from the Hesione simulation without any grid. Symbols, full and dashed lines represent the raw numerical results, the subsequent fit, and the pure coasting trajectory, respectively. 
The radius of the unperturbed interface can be fitted by $R(\tau)=0.0997-79.74 \tau-16370 \tau^{2}+1.16210^{8} \tau^{3}$. At $\tau=350 \mathrm{~ms}$, the distance from a coasting trajectoty is $3 \mathrm{~mm}$ which corresponds to a relative discrepancy for the radius equal to $4 \%$.

The linear growth of a single mode perturbation due to a RT instability in the cylindrical geometry for incompressible fluids has been derived in Ref. [18]. The differential equation for this growth writes as:

$$
\frac{d^{2} a}{d \tau^{2}}+2 \frac{\dot{R}}{R} \frac{d a}{d \tau}-\left(n A t-1 \frac{\ddot{R}}{R}\right) a=0
$$

where $n=2 \pi / \theta_{0}$. This equation is valid in the linear regime i.e. as long as $|n a / R|<1$. The initial conditions of the shot $\# 961$ are $n=24.11, A t=0.687$ and $\nu_{0}=-14.7 \mathrm{~m} / \mathrm{s}$. The resulting growth, $a(t)$, is presented in Fig. 19

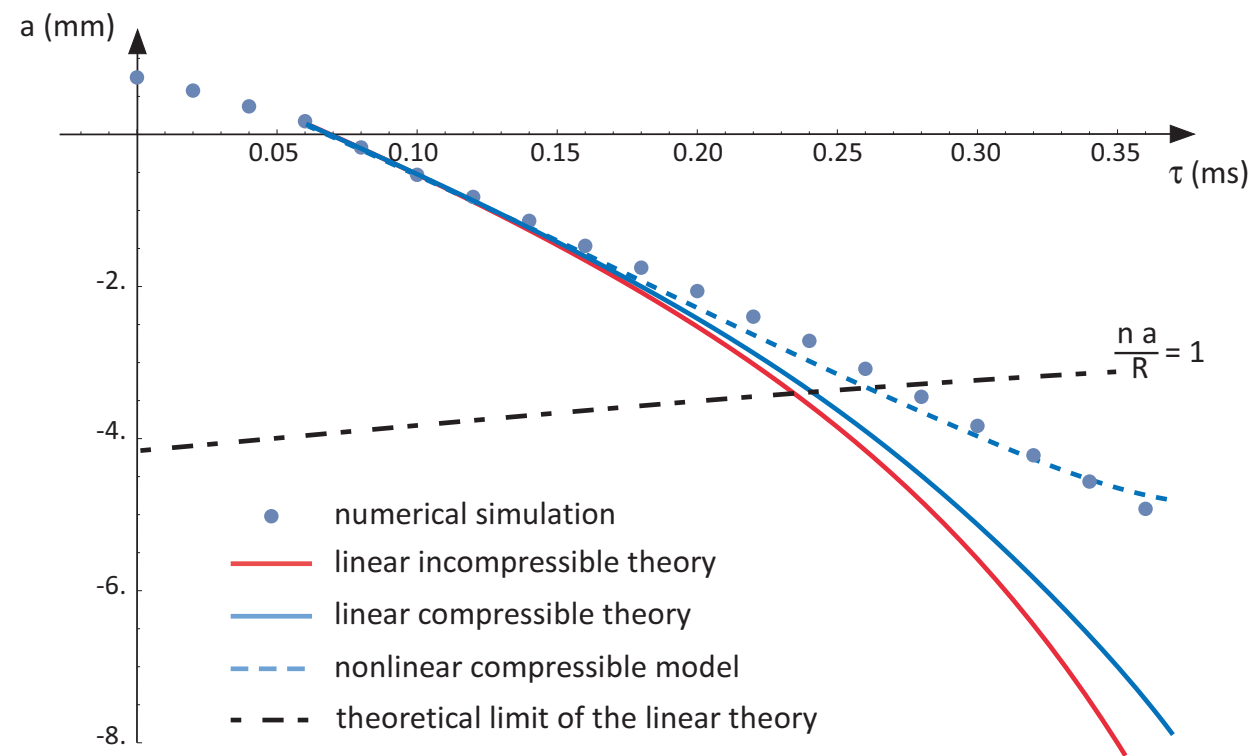

FIG. 19: Growth of the fundamental mode for the initial conditions of shot \#961. Symbols represent the results obtained from the Hesione code. Red and blue curves are obtained from the linear theory for incompressible and compressible fluids. The dot curve is obtained from the nonlinear theory with compressible fluids. The dot-dashed line represents that theoretical range of validity of the linear theory.

The linear theory for incompressible fluids deviates from the numerical simulation around $\tau=0.15 \mathrm{~ms}$ even if the model is theoretically still valid. Two reasons can be invoked in order to explain this discrepancy: the compressibility of the fluids, and the nonlinear regime of the instability.

The effect of compressibility have already been theoretically studied in Refs. [16, 17, 19] by considering uniform compression rate in each fluid, or fixed density profiles. On the contrary, in our case, the implosion is initiated by a collapsing shock wave. The Fig. 20 presents the radial velocities which are obtained from the Hesione code and models using velocity potentials. The latter describe continuously converging flows which are not relevant to our experiments.

In order to estimate the effect of compressibility, we consider time-dependent velocity and density profiles, $v(r, \tau)$ and $\rho(r, \tau)$. However, for the linear analysis, it is enough to know the first order variation of these profiles at the interface. For the velocity, we write $v(r, \tau)=\dot{R}+\beta(\tau)(r-R)$ in each fluid at the interface, and derive the resulting linear analysis for the RT instability in the cylindrical geometry. This derivation takes into account the time and spatial variations of the velocity and density at the interface (see Appendix B). The subsequent differential equation for the amplitude of the perturbation writes as:

$$
\frac{d^{2} a}{d t \tau^{2}}+C_{1}(\tau) \frac{\dot{R}}{R} \frac{d a}{d \tau}-n A t \frac{\ddot{R}}{R} a+C_{2}(\tau) \frac{\dot{R}^{2}}{R^{2}} a=0
$$

The functions $C_{1}(\tau)$ and $C_{2}(\tau)$ are displayed in the Appendix B. 

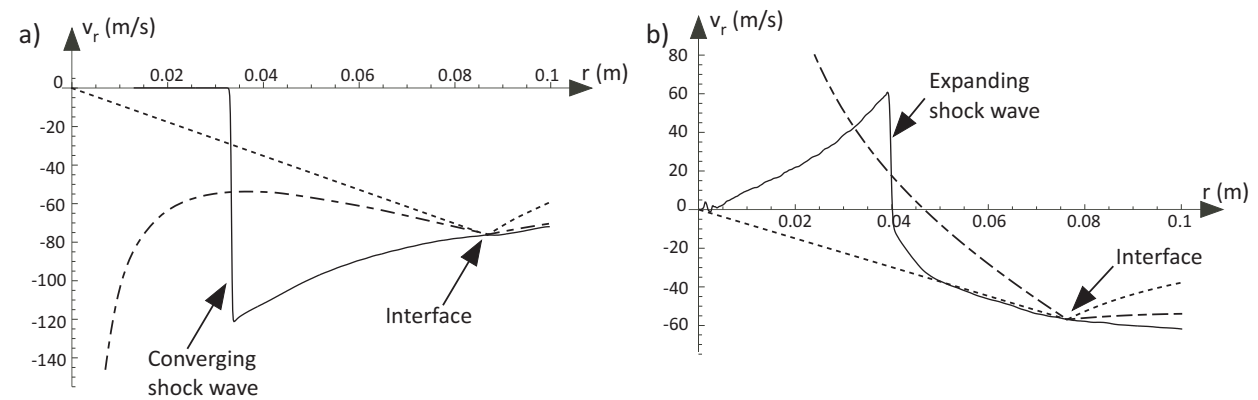

FIG. 20: Radial velocities for shot \#961 from the Hesione code (full line), Amendt's [16] (dotted line) and Epstein's [17] (dash-dotted line) velocity potentials. a- $\tau=0.16 \mathrm{~ms}, \mathrm{~b}-\tau=0.32 \mathrm{~ms}$.

- In Eq.(6), as far as the density is concerned, only the Atwood number is needed. In the studied case, the numerical simulation gives the following fits for the densities in $\mathrm{SF}_{6}$ and air at the interface: $\rho_{S F 6}=8.45+$ $637.42 \tau+2.50710^{6} \tau^{2}$, and $\rho_{\text {air }}=1.55+167.24 \tau+3.368710^{6} \tau^{2}$. The density of the $\mathrm{SF}_{6}$ and the air increases from 8.45 to $11.75 \mathrm{~kg} / \mathrm{m}^{3}$, and from 1.55 to $2 \mathrm{~kg} / \mathrm{m}^{3}$, respectively. However, the time-variation of the Atwood number is small: from 0.687 to 0.706 . As a result, the noticeable increase of the densities at the interface does not play a significant role in the growth of the perturbation.

- The functions $C_{1}(\tau)$ and $C_{2}(\tau)$ depend mainly on the velocity parameter $\beta$. In our case, the time variation of $\beta$ is evaluated from the numerical simulation. Let us remark that if $\beta=-\dot{R} / R$, the Eq.(6) reduces to Eq.(5). This means that if the velocity profile at the interface writes as $v(r, \tau)=\dot{R}-\dot{R} / R(r-R)$, compressible fluids would behave as incompressible ones for the RT instability. The time evolutions of the parameters $\beta(\tau)$, as well as $-\dot{R} / R$ are displayed in Fig. 21 .

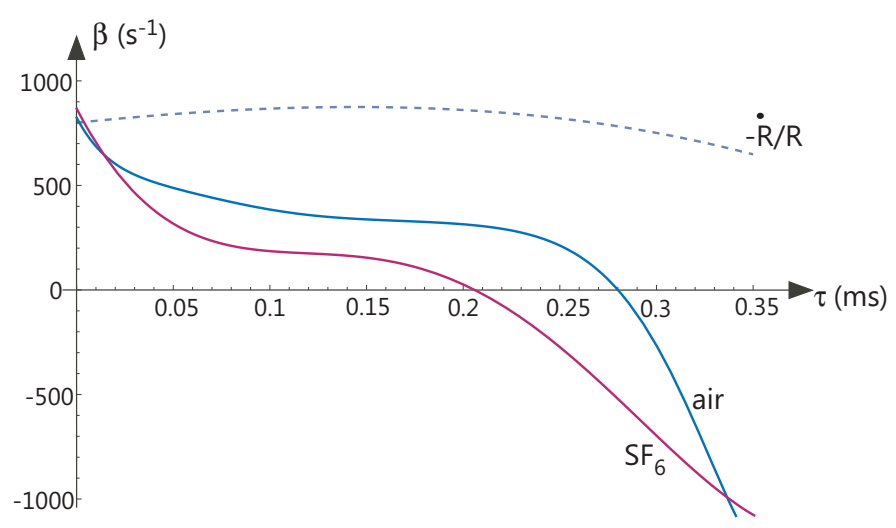

FIG. 21: Parameters $\beta$ at the interface for $\mathrm{SF}_{6}$ and air.

The growth of the perturbation which is predicted by the Eq. (6) is plotted in Fig. 19. Taking into account the compressibility reduces the discrepancy between theory and the numerical simulation, even if the benefit is small. This is remarkable since the values of $\beta$ in $\mathrm{SF}_{6}$ and air clearly depart from $-\dot{R} / R$. The theoretical study of the role of the compressibility through the functions $C_{1}(\tau)$ and $C_{2}(\tau)$, or the parameter $\beta$ is let for future work. In our case, the compressibility gets a small effect on the instability in the linear phase.

In order to estimate the effect of nonlinearity, we start from the weakly nonlinear analysis which is performed in Ref. [23]. This perturbation theory for the RT instability in the cylindrical geometry gives:

$$
\eta_{N L}^{(1)}=\eta_{L}^{(1)}-\frac{1}{R_{0}^{2}}\left[n^{2}\left(3 A^{2}+1\right)-A n-9\right]\left(\eta_{L}^{(1)}\right)^{3}
$$

where $R_{0}=R(0)$, and $\eta^{(1)}$ is the amplitude of the first harmonics. The subscripts $N L$ and $L$ stand for nonlinear and linear, respectively. The radius of convergence of this perturbation theory is small. In order to go beyond the 
singularity, it is common to a use continuation method. From Eq. (7), a $P_{2}^{0}$ Pade approximant is build. It reads:

$$
\eta_{N L}^{(1)}=\frac{\eta_{L}^{(1)}}{1+\frac{1}{R_{0}^{2}}\left[n^{2}\left(3 A^{2}+1\right)-A n-9\right]\left(\eta_{L}^{(1)}\right)^{2}}
$$

The range of validity of Eq.(8) is limited since as the time tends to infinity, this equation predicts that $\eta_{N L}^{(1)}$ tends to 0 . However, it is sufficient to describe the weakly nonlinear regime of the instability. As seen in Fig. 19, the Pade approximant is in good agreement with the numerical simulation. Let us note that the linear amplitude, $\eta_{L}$, which is used to reach such a good agreement comes from the equation for compressible fluids, Eq. (6). The nonlinear growth begins to deviate from the linear theory around $\tau \approx 0.15 \mathrm{~ms}$ which corresponds to $|n a / R| \approx 0.5$.

To summarize our analysis of the studied RM-RT instability in the cylindrical geometry:

- from $\tau=0$ to $0.15 \mathrm{~ms}$, which corresponds to $r / R_{0}=1$ to 0.88 and $|n a / r|=0.17$ to 0.41 , the instability is in the linear regime and the compressible effects are negligible. Furthermore, as comparison with pure RM theory shows, the instability still does not feel any RT effect.

- from $\tau=0.15$ to $0.17 \mathrm{~ms}$, which corresponds to $r / R_{0}=0.88$ to 0.86 and $|n a / r|=0.41$ to 0.47 , the compressibility must be taken into account. In our $\mathrm{SF}_{6}$ /air configuration, the compressibility reduces the growth of the instability. The deceleration of the interface begins to influence the growth rate. The interface dynamics is driven by both RM and RT instabilities.

- from $\tau=0.17$ to $0.36 \mathrm{~ms}$, which corresponds to $r / R_{0}=0.86$ to 0.74 and $|n a / r|=0.47$ to 1.54 , the instability is in the nonlinear regime, and even if its effect is small, the compressibility must be taken into account.

- In the cylindrical geometry, and for the studied convergence ratio, the saturation of the growth of the instability in its nonlinear regime is characterized by a linear time variation.

\section{CONCLUDING REMARKS}

This study has shown that in the cylindrical geometry the RM instability can transform into the RT instability. A linear time dependent growth can be obtained in the nonlinear regim. We underline several points about hydrodynamic instabilities in converging geometry:

- The motion of the background flow must be carefully analyzed in order to check if any deceleration occurs. In our case, as the interface is not purely coasting. A relative discrepancy equal to $4 \%$ about the radius has led to the disappearance of the expected classical RM logaritmic saturation. Another example of this apparent postponement of the saturation of the RM instability can be found in laser experiments [6]. The authors postulate that this lack of saturation is due to the suppression of the growth of secondary instabilities due to the convergent effects. However, the cylindrical shell of these experiments undergoes a deceleration [37] which was discarded by the authors. This deceleration leads to a $25 \%$ relative discrepancy between the actual and the coasting trajectories. In the light of our study, these data could be reconsider to check if the postponent of the saturation is due to the RT instability.

- The Eq.(8) succeeds to describe the weakly nonlinear growth of the RM+RT instability. However, the range of validity of such a Pade approximant is quite limited. In order to predict the nonlinear growth of the instability, theories without secular terms are needed. Our data provide a good test case for candidate theories in the weakly nonlinear regime. For higher convergence ratio, it would be interesting to see if the growth remains linear time dependent.

- In order to study the compressibility effects in ICF relevant experiments, the influence of the shock waves on the background flow must be taken into account. Collapsing shock waves must be consider as a singular limit condition for the velocity. Furthermore, they create a stratified fluid in their wake. If velocity potentials are to be used to study the convergent RT instability seeded by RM instability, they must adequately describe such a flow. Our study has shown that the spatial slope of $v_{r}(r, t), \beta(t)$, influences the growth of the instability. With a relevant velocity potential, the Eq.(6) will be a useful tool to theoretically study when the compressibility comes into play. 


\section{Appendix A: The onset of a reflected shock wave at the interface 2}

In this section, the origin of time is such that the rebounding shock waves on the grid bars would have a zero radius at $t=0$. In order to explain the onset of a reflected shock wave at the second interface, the grid which holds the nitrocellulosic membrane must be taken into account. The bars of the grid are seen by the incident shock wave as an immovable wall. As a result, the shock wave locally bounces back on the bars. In between the latter, normal refraction occurs. The velocity of the reflected shock wave can be estimated [28], and equals to $111.9 \mathrm{~m} / \mathrm{s}$. The bars of the grid are cylinders which diameter is $\Phi_{b a r}=1 \mathrm{~mm}$. The reflection of the shock wave on the bars generates an cylindrical high pressure area around each bar. As a consequence, the interaction between the shock wave and the grid creates an array of cylindrical expanding shock waves which are centered on each bar. Their initial diameters equal to $\Phi_{b a r}$. We note, $r_{\text {local }}$, the radius of each shock wave centered on each bar. Following Whitham and Guderley [28, 35], we can estimate the trajectory, $r_{\text {local }}(t)$, of an expanding shock wave:

$$
r_{\text {local }}(t)=(C t)^{n}
$$

where $n=\frac{n_{c}}{1+n_{c}}, n_{c}=1+\frac{2}{\gamma}+\sqrt{\frac{2 \gamma}{\gamma-1}}[28,36]$, and $C$ a constant. In $\mathrm{SF}_{6}, n$ equals to 0.8858 . In order to compute $C$, we use the initial conditions:

$$
\left\{\begin{array}{l}
r_{\text {local }}\left(t_{0}\right)=\left(C t_{0}\right)^{n}=\frac{\Phi_{\text {bar }}}{2} \\
\dot{r}_{\text {local }}\left(t_{0}\right)=n C^{n} t_{0}^{n-1}=111.9
\end{array}\right.
$$

The duration $t_{0}$ can be seen as the time which would be needed by an hypothetical cylindrical expanding shock wave generated at $r_{\text {local }}=0$ to reach $r_{\text {local }}=\Phi_{\text {bar }} / 2$. Equations (A2) lead to the following solution: $t_{0}=3.958 \mu \mathrm{s}$, and $C=47.41$. The bars are $d=1.4 \mathrm{~cm}$ away from each other. When the expanding shock waves have filled the gap between bars, they coalesce. The resulting shock wave is now centered on the apex, and its velocity stabilizes. Going from the local reference frame centered on each bar to the reference frame centered on the apex of the shock tube, we write:

$$
r_{r}^{(2)}(t) \approx r_{\text {local }}(t)+0.1
$$

The theoretical velocity of the expanding shock wave from the slowdown phase (Eq.(A1)) to the constant velocity phase $\left(r_{\text {local }}=d / 2 \Rightarrow W_{r}^{(2)}=\dot{r}_{r}^{(2)}=77.96 \mathrm{~m} / \mathrm{s}\right)$ is presented on Fig. 22-a.
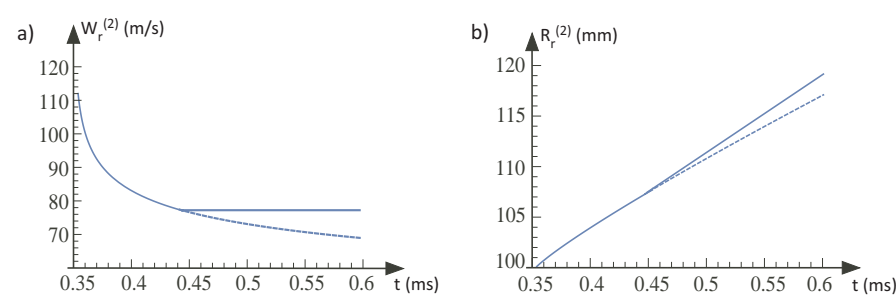

FIG. 22: Reflected shock wave on the grid bar at the interface 2. a- Velocity. b- Trajectory. Full, and dashed lines represent the expansion and the coalescence phases, respectively.

The resulting trajectory, $r_{r}^{(2)}(t)$, is plotted in Fig. 22-b. It is in reasonable agreement with the experimental data: at $t=0.6 \mathrm{~ms}$, the radius of the reflected shock wave is estimated at $122 \mathrm{~mm}$ in the experiment, and $119 \mathrm{~mm}$ by the theory. In order to assess our conclusions, eulerian numerical simulations with the Hesione code were run in the planar geometry. These numerical simulations are solely devoted to the shock-interface interaction in the presence of bars. As seen in Fig. 23, at $t=5 \mu \mathrm{s}$, high pressure area are created on the bars. For approximatively $90 \mu \mathrm{s}$, the resulting shock waves expand. Afterwards, they coalesce in a single wave.

In conclusion, the reflected wave at the second interface is a mix between shock waves at the bar locations, and classical rarefaction in between. After expanding, the shock waves eventually coalesce, and overtake the rarefaction waves. 

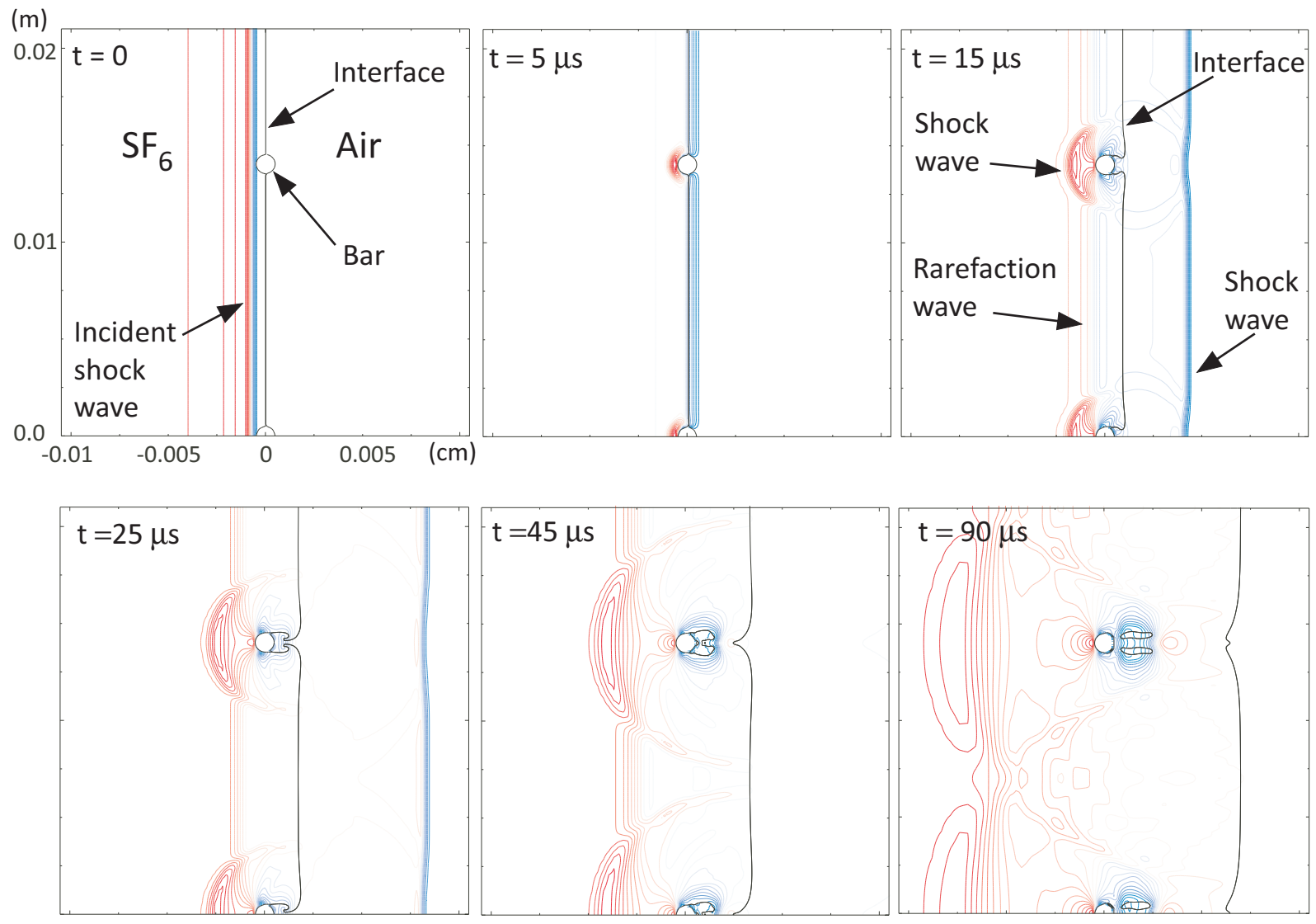

FIG. 23: Interaction between a planar shock wave, an interface $\mathrm{SF}_{6} /$ air, and the grid bars. 


\section{Appendix B: Linear theory for the RT instability in cylindrical geometry and compressible fluids}

The equation of momentum conservation for compressible fluids writes as:

$$
\frac{\partial \vec{v}}{\partial t}+(\vec{v} \cdot \nabla) \vec{v}=-\frac{1}{\rho} \vec{\nabla} P+\vec{g} \text { with } \rho(r, t)
$$

For incompressible fluids, $\vec{v}=-\vec{\nabla} \Phi$ and $\Delta \Phi=0$ gives the form of $\Phi$. For compressible fluids, it is always possible to postulate $\vec{v}=-\vec{\nabla} \Phi$ even if the expression for $\Phi$ is unknown. However, following Epstein and Amendt [16, 17], we can assume that the perturbations are incompressible. As a result, let the potentials for the fluid near the apex, and the fluid far from the apex write as:

$$
\begin{aligned}
& \Phi^{-}=\Phi_{0}^{-}+b_{l}^{-} r^{-l} \cos (l \theta) \\
& \Phi^{+}=\Phi_{0}^{+}+b_{l}^{+} r^{+l} \cos (l \theta)
\end{aligned}
$$

where $\Phi_{-}^{0}$ and $\Phi_{+}^{0}$ are the potentials for the unperturbed flow. For the unpertubed flow, at the interface $\eta=R$, Bernoulli's equations writes as:

$$
\left\{\begin{array}{l}
\rho_{0}^{-}\left(\frac{\partial \Phi_{0}^{-}}{\partial t}-\frac{1}{2} \dot{R}^{2}-g \eta\right)+P_{0}^{-}=C^{-} \\
\rho_{0}^{+}\left(\frac{\partial \Phi_{0}^{+}}{\partial t}-\frac{1}{2} \dot{R}^{2}-g \eta\right)+P_{0}^{+}=C^{+}
\end{array}\right.
$$

We now consider the perturbed flow:

$$
\left\{\begin{array}{l}
\rho^{-}\left(\frac{\partial \Phi^{-}}{\partial t}-\frac{1}{2}\left(v_{r}^{-}\right)^{2}-g \eta\right)+P_{0}^{-}+\delta P^{-}=C^{-} \\
\rho^{+}\left(\frac{\partial \Phi^{+}}{\partial t}-\frac{1}{2}\left(v_{r}^{+}\right)^{2}-g \eta\right)+P_{0}^{+}+\delta P^{+}=C^{+}
\end{array}\right.
$$

We have:

$$
\begin{aligned}
& v_{r}^{-}=\dot{R}-l b_{l}^{-} r^{-l-1} \cos (l \theta) \\
& v_{r}^{+}=\dot{R}+l b_{l}^{+} r^{+l-1} \cos (l \theta)
\end{aligned}
$$

Substituting Eq.(B5) in Eq.(B4), and using Eq.(B3), we linearize the equations by considering that $\eta=\dot{R}+a \cos (l \theta)$, $a / R<<1$, and $\delta P^{-}=\delta P^{+}$at the interface:

$$
\rho_{0}^{-}(t)\left(\dot{b}_{l}^{-} r^{-l}+\dot{R} l b_{l}^{-} r^{-l-1}-g a\right)=\rho_{0}^{+}(t)\left(\dot{b}_{l}^{+} r^{+l}-\dot{R} l b_{l}^{+} r^{+l-1}-g a\right)
$$

Let us now study the equation of motion of the interface.

If an incompressible fluid is considered, following Mikaelian [18], we write $\Phi=-R \dot{R} \ln (r), v_{r}=-\vec{\nabla} \Phi=\dot{R}-\frac{\dot{R} a}{R} \cos (l \theta)$ at the interface. Furthermore, from the definition of $\eta$, we also have $v_{r}=\dot{R}+\dot{a} \cos (l \theta)$. Equating these two expressions for $v_{r}$ leads to the following differential equations for each fluid:

$$
-\frac{\dot{R} a}{R} \pm l b_{l}^{\mp} r^{\mp l-1}=\dot{a}
$$

For compressible fluids, in the vicinity of the interface, it is always possible to write:

$$
v_{r}^{\mp}=\dot{R}+\beta^{\mp}(t)(r-R)
$$

Equating the previous equation with $v_{r}=\dot{R}+\dot{a} \cos (l \theta)$ gives:

$$
\beta^{\mp} a \pm l b_{l}^{\mp} r^{\mp l-1}=\dot{a}
$$

If $\beta^{\mp}=-\frac{\dot{R}}{R}$, the equations for the compressible fluids are similar to the ones for the incompressible ones. After substitution of Eqs.(B9) in Eq.(B6) and some cumbersome calculations, the following differential equation is obtained for the linear growth of the amplitude $a(t)$ :

$$
\ddot{a}-l \frac{\ddot{R} a}{R} A t(t)+\frac{\dot{a} \dot{R}}{R} C_{1}-\frac{\dot{R}^{2}}{R^{2}} a C_{2}=0
$$


where $C_{1}=\frac{\rho^{+}(t)\left(1-\frac{R}{R} \beta^{+}\right)+\rho^{-}(t)\left(1-\frac{R}{\dot{R}} \beta^{-}\right)}{\rho^{+}+\rho^{-}}$and $C_{2}=\frac{\rho^{+}(t)\left(\frac{R}{\dot{R}} \beta^{+}+\frac{R^{2}}{\dot{R}^{2}} \dot{\beta}^{+}\right)+\rho^{-}(t)\left(\frac{R}{\dot{R}} \beta^{-}+\frac{R^{2}}{\dot{R}^{2}} \dot{\beta}^{-}\right)}{\rho^{+}(t)+\rho^{-}(t)}$.

[1] R.D. Richtmyer, "Taylor instability in shock acceleration of compressible fluids," Commun. Pure Appl. Math. 13, 297 (1960).

[2] E.E. Meshkov, "Interface of two gases accelerated by a shock wave," Fluid Dyn. 4, 101 (1969).

[3] X. Luo, J. Ding, M. Wang, Z. Zhai, and T. Si, "A semi-annular shock tube for studying cylindrically converging RichtmyerMeshkov instability", Phys. Fluids 27, 091702 (2015).

[4] D.L. Tubbs et al., "Cylindrical implosion experiments using laser direct drive", Phys. Plasmas 6, 2095 (1999).

[5] S.G. Glendinning et al., "Ablation front Rayleigh-Taylor growth experiments in spherically convergent geometry", Phys. Plasmas 7, 2033 (2000).

[6] J.R. Fincke, N.E. Lanier, S.H. Batha, R.M. Hueckstaedt, G.R. Magelssen, S.D. Rothman, K.W. Parker, and C.J. Horsfield "Postponement of saturation of the Richtmyer-Meshkov instability in a convergent geometry", Phys. Rev. Lett. 93, 115003 (2004).

[7] V.A. Smalyuk et al., "First measurements of hydrodynamic instability growth in indirectly driven implosions at ignitionrelevant conditions on the National Ignition Facility", Phys. Rev. Lett. 112, 185003 (2014).

[8] E.M. Giraldez et al., "Machining of two-dimensional sinusoidal defects on ignition-type capsules to study hydrodynamic instability at the National Ignition Facility", Fusion Sci. Tech. 70, 258 (2016).

[9] K.S. Raman et al., "An in-flight radiography platform to measure hydrodynamic instability growth in inertial confinement fusion capsules at the National Ignition Facility", Phys. Plasmas 21, 072710 (2014).

[10] E.M. Campbell et al., "Nova experimental facility", Rev. Sci. Instrum. 57, 2101 (1986).

[11] T. Boehly et al., "Initial performance of the OMEGA laser system", Opt. Commun. 133, 495 (1997).

[12] G.H. Miller, E.I. Moses, and C.R. Wuest, "The National Ignition Facility: enabling fusion ignition for the 21st century", Nucl. Fusion 44, S228 (2004).

[13] J.L. Peterson, D.T. Casey, O.A. Hurricane, K.S. Ramand, H.F. Robey, and V.A. Smalyuk, "Validating hydrodynamic growth in National Igntion Facility implosions", Phys. Plasmas 22, 056309 (2015).

[14] G.I. Bell, "Taylor instability on cylinders and spheres in small amplitude approximation," Los Alamos Scientific Laboratory Report No. LA-1321, 1951.

[15] M.S. Plesset, "On the stability of fluid flows with spherical symmetry," J. Appl. Phys. 25, 96 (1954).

[16] P. Amendt, J.D. Colvin, J.D. Ramshaw, H.F. Robey, and O.L. Landen, "Modified Bell-Plesset effect with compressibility: application to double-shell ignition target designs," Phys. Plasmas 10, 820 (2003).

[17] R. Epstein, "On the Bell-Plesset effects: the effects of uniform compression and geometrical convergence on the classical Rayleigh-Taylor instability," Phys. Plasmas 11, 5114 (2004).

[18] K.O. Mikaelian, "Rayleigh-Taylor and Richtmyer-Meshkov instabilities and mixing in statified cylindrical shells," Phys. Fluids 17, 094105 (2005).

[19] H. Yu, and D. Livescu, "Rayleigh-Taylor instability in cylindrical geometry with compressible fluids," Phys. Fluids 20, 104103 (2008).

[20] M. Lombardini, and D.I. Pullin, "Small-amplitde perturbations in the three-dimensional cylindrical Richtmyer-Meshkov instability," Phys. Fluids 21, 114103 (2009).

[21] Lord Rayleigh, "Investigation of the character of the equilibrium of an incompressible heavy fluid of variable density," Proc. London Math. Soc. 14, 170 (1882).

[22] G.I. Taylor, "The instability of liquid surfaces when accelerated in a direction perpendicular to their planes," Proc. R. Soc. London Ser A 201, 192 (1950).

[23] L.F. Wang, J.F. Wu, W.H. Ye, W.Y. Zhang, and X. T. He, "Weakly nonlinear incompressible Rayleigh-Taylor instability growth at cylindrically convergent interfaces," Phys. Plasmas 20, 042708 (2013).

[24] L. Biamino, C. Mariani, G. Jourdan, L. Houas, M. Vandenboomgaerde, and D. Souffland, "Planar shock focusing through perfect gas lens: first experimental demonstration," J. Fluids Eng. 136(9), 091204 (2014).

[25] L. Biamino, G. Jourdan, C. Mariani, L. Houas, M. Vandenboomgaerde, and D. Souffland, "On the possibility of studying the converging Richtmyer-Meshkov instability in a conventional shock tube," Exp. Fluids 56:26 (2015).

[26] M. Vandenboomgaerde, and C. Aymard, "Analytical theory for planar shock focusing through perfect gas lens and shock tube experiment designs," Phys. Fluids 23, 016101 (2011).

[27] G. Jourdan, L. Houas, L. Schwaederlé, G. Layes, R. Carrey, and F. Diaz, "A new variable inclination shock tube for multiple investigations,", Shock Waves, 13(6), 501 (2004).

[28] G.B. Whitham, Linear and Nonlinear Waves (Wiley-Interscience, New York, 1974).

[29] D. Eder, K. Koniges, F. Bonneau, J. Vierne, P. Combis, J. Andrews,K. Mann, and B. MacGowan, 'Simulation of shrapnel to aid in the design of NIF/LMJ target diagnostic configurations," Proceedings of Inertial Fusion Science and Applications (IFSA-2003) (Elsevier, New York, 2004), pp. 572-575.

[30] F. Bonneau, P. Combis, J.L. Rullier, J. Vierne, B. Bertussi, M. Commandre, L. Gallais, J. Y. Natoli, I. Bertron, F. Malaise, and J. T. Donohue, "Numerical simulations for description of UV laser interaction with gold nanoparticles embedded in silica," Appl. Phys. B: Lasers Opt. 78, 447 (2204).

[31] C. Mariani, M. Vandenboomgaerde, G. Jourdan, D. Souffland and L. Houas, "Investigation of the Richtmyer-Meshkov 
Instability with Stereolithographed Interfaces", Phys. Rev. Lett. 100, 254503 (2008).

[32] K.O. Mikaelian, "Explicit expressions for the evolution of single-mode Rayleigh-Taylor and Richtmyer-Meshkov instabilities at arbitrary Atwood numbers," Phys. Rev. E 67, 026319 (2003).

[33] W.H. Liu, C.P. Yu, W.H. Ye, L.F. Wang, and X.T. He, "Nonlinear theory of classical cylindrical Richtmyer-Meshkov instability for arbitrary Atwood numbers," Phys. Plasmas 21, 062119 (2014).

[34] M. Vandenboomgaerde, D. Souffland, C. Mariani, L. Biamino, G. Jourdan, and L. Houas, "An experimental and numerical investigation of the dependency on the initial conditions of the Richtmyer-Meshkov instability," Phys. Fluids 26, 024109 (2014).

[35] G. Guderley, "Starke kugelige und zylindrische Verdichtungsstöße in der Nähe des Kugelmittelpunker bzw. der Zilinderachse," Luftfahrforsch 19, 302 (1942).

[36] R.F. Chisnell, "The motion of a shock wave in a channel, with application to cylindrical and spherical shocks," J. Fluid Mech. 2, 286 (1957).

[37] N.E. Lanier, C.W. Barnes, S.H. Batha, R.D. Day, G.R. Magelssen, J.M. Scott, A.M. Dunne, K.W. Parker, and S.D. Rothman, "Multimode seeded Richtmyer-Meshkov mixing in a convergent, compressible, miscible plasma system," Phys. Plasmas, 10, 1816 (2003). 